\title{
Rapid Assembly of the Polyhydroxylated $\beta$-Amino Acid Constituents of Microsclerodermins C, D and E
}

Thomas Hjelmgaard, ${ }^{\dagger}$ Sophie Faure, ${ }^{\dagger}$ Pascale Lemoine, ${ }^{\ddagger}{ }^{\star}$ Bernard Viossat, ${ }^{\ddagger}$ and David J. Aitken ${ }^{\xi, *}$

${ }^{\dagger}$ Université Blaise Pascal - Clermont-Ferrand 2.

${ }^{\ddagger}$ Université René Descartes - Paris 5.

${ }^{\S}$ Université Paris-Sud 11.

\section{CONTENTS}

S2: General experimental methods and comments

S3: Synthesis of phosphorus reagents S1 and 8

S4: Optimization tables for the synthesis of alcohols $\mathbf{2}$ and $\mathbf{9}$

S5-S13: Expermental procedures and chacaracterization data for all intermediates and products.

S15-S32: ${ }^{1} \mathrm{H}$ and ${ }^{13} \mathrm{C}-\mathrm{NMR}$ of all intermediates and products

S33: Crystallographic data for $\mathbf{7 b}$ and $\mathbf{7 c}$

S35: References 
THF was distilled under $\mathrm{N}_{2}$ from potassium/benzophenone. $\mathrm{CH}_{2} \mathrm{Cl}_{2}$ was distilled under $\mathrm{N}_{2}$ from $\mathrm{CaH}_{2}$. Benzene was dried over sodium. EtOAc and cyclohexane for column chromatography were distilled before use. $\mathrm{MeOH}$ was distilled under $\mathrm{N}_{2}$ from $\mathrm{CaH}_{2}$ and stored over $4 \AA$ molecular sieves. Diethyl allylphosphonate, diethyl benzylphosphonate, DMSO and $\mathrm{Et}_{3} \mathrm{~N}$ were dried over $4 \AA$ molecular sieves. Ti(OEt) $)_{4}$ (Acros, tech. grade, 85\%), and all other solvents and chemicals obtained from commercial sources were, unless otherwise stated, used without further purification.

Melting points were determined on a Reichert microscope apparatus and are uncorrected. Specific rotations were measured on a Jasco DIP-370 polarimeter using a $10 \mathrm{~cm}$ cell. NMR spectra were recorded on a $400 \mathrm{MHz}$ Bruker AC 400 spectrometer. Chemical shifts are referenced to the residual solvent peak and $J$ values are given in $\mathrm{Hz}$. The following multiplicity abbreviations are used: (s) singlet, (d) doublet, (t) triplet, (q) quartet, (m) multiplet, and (br) broad. Where applicable, assignments were based on HSQC, COSY and/or $J$-mod-experiments: (-) designates a (C) or a $\left(\mathrm{CH}_{2}\right) ;(+)$ designates a $(\mathrm{CH})$ or a $\left(\mathrm{CH}_{3}\right)$. TLC was performed on Merck TLC aluminum sheets, silicagel $60, \mathrm{~F}_{254}$. Progression of reactions was, when applicable, followed by NMR and/or TLC. Visualizing of spots was effected with UV-light and/or ninhydrin in EtOH/AcOH. Flash chromatography was performed with Merck silica gel 60, 40-63 $\mu \mathrm{m}$. Unless otherwise stated, flash chromatography was performed in the eluent system for which the $R_{f}$ values are given. HRMS were recorded on a Micromass Q-Tof Micro (3000V) apparatus.

As a general comment, we recommend that particuliar care be taken when selecting and purchasing the specific enantiomerically enriched sulfinamides, as we have found that naming and drawing errors are not uncommon both in both scientific and manufacturers' literature. 
The nucleophilic phosphorus reagents used in the synthesis of protected AETD 13, phosphonate S1 and phosphonium bromide 8, were prepared as follows (Scheme S1). Heck coupling of 4iodophenetole with diethyl allylphosphate following an adapted literature procedure provided phosphonate $\mathbf{S 1}$ in 51\% yield, ${ }^{\mathrm{S} 1}$ while phosphonium bromide 8 was obtained (as a THF complex) in a two step sequence by reduction of ethyl trans-4-ethoxycinnamate with DIBAL-H, ${ }^{\mathrm{S} 2}$ followed by reaction with $\mathrm{PPh}_{3} / N$-bromosuccinimide ${ }^{\mathrm{S} 3}$ in $74 \%$ overall yield.

Scheme S1. Synthesis of Wittig-type reagents S1 and 8<smiles>C=CC[P+]=CCc1ccc(OCC)cc1</smiles><smiles>CCOC(=O)/C=C/c1ccc(OCC)cc1</smiles>

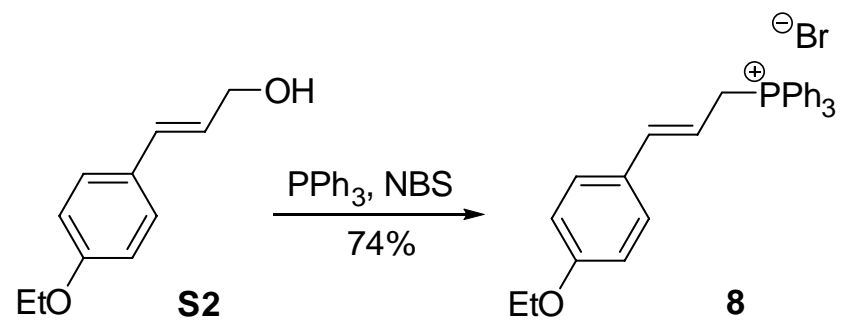

NBS $=N$-bromo-succinimide. 
Table S1. Optimization of synthesis of alcohol 2.

\begin{tabular}{|c|c|c|c|c|}
\hline Entry & $\mathbf{R}(\mathbf{e q})^{a}$ & Temp. $\left({ }^{\circ} \mathrm{C}\right)$ & Yield (\%) & $\boldsymbol{E} / \mathbf{Z}^{b}$ \\
\hline 1 & $\mathrm{I}^{-} \mathrm{Ph}_{3} \mathrm{P}^{+}(3.0)$ & $0 \rightarrow \mathrm{rt}$ & 58 & $58: 42$ \\
\hline 2 & $(\mathrm{EtO})_{2} \mathrm{P}(\mathrm{O})(3.0)$ & $0 \rightarrow \mathrm{rt}$ & 23 & $>99: 1^{c}$ \\
\hline 3 & $(\mathrm{EtO})_{2} \mathrm{P}(\mathrm{O})(5.0)$ & $0 \rightarrow \mathrm{rt}$ & 7 & $>99: 1^{c}$ \\
\hline 4 & $(\mathrm{EtO})_{2} \mathrm{P}(\mathrm{O})(3.0)$ & $0 \rightarrow 45$ & 39 & $>99: 1^{c}$ \\
\hline 5 & $(\mathrm{EtO})_{2} \mathrm{P}(\mathrm{O})(3.0)$ & $0 \rightarrow$ reflux & 38 & $>99: 1^{c}$ \\
\hline 6 & $(\mathrm{EtO})_{2} \mathrm{P}(\mathrm{O})(2.0)$ & $0 \rightarrow 45$ & 52 & $>99: 1^{c}$ \\
\hline 7 & $(\mathrm{EtO})_{2} \mathrm{P}(\mathrm{O})(1.5)$ & $0 \rightarrow 45$ & 13 & $>99: 1^{c}$ \\
\hline
\end{tabular}

${ }^{a}$ Equimolar amounts of ${ }^{n} \mathrm{BuLi}$ used. ${ }^{b}$ As judged by NMR. ${ }^{c}$ Only one isomer visible on NMR.

Table S2. Optimization of synthesis of alcohol 9.
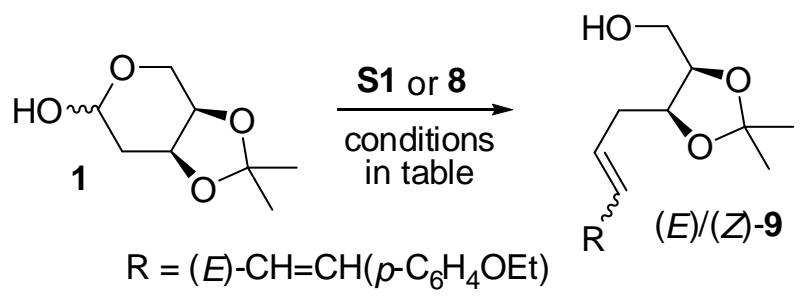

\begin{tabular}{ccccc}
\hline Entry & Conditions $^{a}$ & Temp. $\left({ }^{\circ} \mathbf{C}\right)$ & Yield (\%) & E/Z \\
\hline 1 & $\mathbf{S 1} /{ }^{n} \mathrm{BuLi} / \mathrm{THF}$ & $0 \rightarrow 45$ & trace & - \\
2 & $\mathbf{S 1} /{ }^{n} \mathrm{BuLi} / \mathrm{THF}$ & $0 \rightarrow \mathrm{reflux}$ & trace & - \\
3 & $\mathrm{~S} 1 /{ }^{t} \mathrm{BuOK} / \mathrm{THF}$ & $\mathrm{rt}$ & 0 & - \\
4 & $\mathrm{~S} 1 / \mathrm{KHMDS} /$ benzene & $0 \rightarrow$ reflux & 0 & \\
5 & $\mathbf{8} /{ }^{n} \mathrm{BuLi} / \mathrm{THF}$ & $0 \rightarrow \mathrm{rt}$ & 0 & - \\
6 & $\mathbf{8} /{ }^{n} \mathrm{BuLi} / \mathrm{THF}$ & $0 \rightarrow$ reflux & 0 & - \\
7 & $\mathbf{8} / \mathrm{KHMDS} /$ benzene & $0 \rightarrow \mathrm{rt}$ & 68 & $72: 28^{b}$
\end{tabular}

${ }^{a} 2.0$ eq. phosphorus reagent and 2.0 eq. base used in every case. ${ }^{b} E$ and $Z$ isomers separated by column chromatography. 


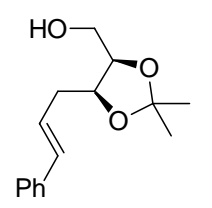

\{(4R,5S)-2,2-Dimethyl-5-[(E)-3-phenylallyl]-1,3-dioxolan-4-yl\}methanol (2). To a solution of diethyl benzylphosphonate $(3.60 \mathrm{~mL}, 17.2 \mathrm{mmol})$ in THF $(50 \mathrm{~mL})$ at 0 ${ }^{\circ} \mathrm{C}$ under Ar was added 1.6 $\mathrm{M}{ }^{n} \mathrm{BuLi}$ in hexanes $(10.75 \mathrm{~mL}, 17.2 \mathrm{mmol})$ and the mixture was stirred for $20 \mathrm{~min}$. A solution of $\mathbf{1}^{\mathrm{S} 4}(1.51 \mathrm{~g}, 8.66 \mathrm{mmol})$ in THF (15 $\mathrm{mL}$ ) was then added dropwise and the mixture was stirred for $19 \mathrm{~h}$ at approx. $45^{\circ} \mathrm{C}$. The reaction was quenched by addition of satd. aq. $\mathrm{NH}_{4} \mathrm{Cl}(55 \mathrm{~mL})$. The mixture was diluted with water (55 $\mathrm{mL})$ and EtOAc $(140 \mathrm{~mL})$ and the organic phase was isolated. The aqueous phase was extracted with EtOAc $(2 \times 140 \mathrm{~mL})$ and the combined organic phases were dried over $\mathrm{MgSO}_{4}$, filtered, concentrated and dried in vacuo. Flash chromatography of the residue yielded $2(1.12 \mathrm{~g}, 52 \%)$ as a yellowish oil: $R_{f}($ cyclohexane/EtOAc $60: 40)=0.41 ;[\alpha]^{22}{ }_{\mathrm{D}}=-15.7\left(c 0.63, \mathrm{CHCl}_{3}\right) ;{ }^{1} \mathrm{H} \mathrm{NMR}$ (400 MHz, $\left.\mathrm{CDCl}_{3}\right): \delta 7.38-7.18(5 \mathrm{H}, \mathrm{m}), 6.49(1 \mathrm{H}, \mathrm{d}, J=15.9 \mathrm{~Hz}), 6.23(1 \mathrm{H}$, ddd, $J=15.9,7.6$, $6.3 \mathrm{~Hz}), 4.31(1 \mathrm{H}, \mathrm{ddd}, J=8.1,5.8,5.8 \mathrm{~Hz}), 4.22(1 \mathrm{H}, \mathrm{ddd}, J=5.8,5.8,5.8 \mathrm{~Hz}), 3.70(2 \mathrm{H}, \mathrm{dd}, J$ $=5.8,5.8 \mathrm{~Hz}), 2.61-2.52(1 \mathrm{H}, \mathrm{m}), 2.49-2.40(1 \mathrm{H}, \mathrm{m}), 1.95(1 \mathrm{H}, \mathrm{t}, J=5.8 \mathrm{~Hz}), 1.51(3 \mathrm{H}, \mathrm{s}), 1.39$ $(3 \mathrm{H}, \mathrm{s}) ;{ }^{13} \mathrm{C} \mathrm{NMR}\left(100 \mathrm{MHz}, \mathrm{CDCl}_{3}\right): \delta 137.1(-), 132.4(+), 128.5(2 \mathrm{C},+), 127.3(+), 126.1(2 \mathrm{C}$, +), $125.8(+), 108.3(-), 77.8(+), 76.5(+), 61.7(-), 33.0(-), 28.1(+), 25.4(+)$; HRMS (TOF MS $\mathrm{ES}^{+}$) calcd for $\mathrm{C}_{15} \mathrm{H}_{20} \mathrm{O}_{3} \mathrm{Na}[\mathrm{M}+\mathrm{Na}]^{+} m / z$ 271.1305, found 271.1312 .

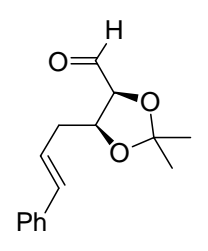

(4S,5S)-2,2-Dimethyl-5-[(E)-3-phenylallyl]-1,3-dioxolane-4-carbaldehyde (3). To a solution of DMSO $(1.29 \mathrm{~mL}, 18.2 \mathrm{mmol})$ in $\mathrm{CH}_{2} \mathrm{Cl}_{2}(25 \mathrm{~mL})$ at $-78{ }^{\circ} \mathrm{C}$ under $\mathrm{Ar}$ was added dropwise oxalyl chloride $(0.79 \mathrm{~mL}, 9.06 \mathrm{mmol})$. After $10 \mathrm{~min}$ a solution of $2(1.75 \mathrm{~g}, 7.05 \mathrm{mmol})$ in $\mathrm{CH}_{2} \mathrm{Cl}_{2}(40 \mathrm{~mL})$ was added over $5 \mathrm{~min}$ and stirring was continued for $1.5 \mathrm{~h}$. $\mathrm{Et}_{3} \mathrm{~N}(5.0 \mathrm{~mL}, 35.9 \mathrm{mmol})$ was then added and the mixture was allowed to heat to $-5^{\circ} \mathrm{C}$ over $2.5 \mathrm{~h}$. The reaction mixture was poured into ice water $(55 \mathrm{~mL}$ ice cold water + $20 \mathrm{~g}$ ice) and the organic phase was isolated. The aqueous phase was extracted with $\mathrm{CH}_{2} \mathrm{Cl}_{2}$ $(2 \times 75 \mathrm{~mL})$, and the combined organic phases were washed with brine $(30 \mathrm{~mL})$, dried over $\mathrm{MgSO}_{4}$, filtered, concentrated and dried in vacuo. Flash chromatography of the residue yielded 3 $(1.48 \mathrm{~g}, 85 \%)$ as a pale yellowish oil: $R_{f}($ cyclohexane/EtOAc $75: 25)=0.41 ;[\alpha]^{22}{ }_{\mathrm{D}}=-45.8(c$ 0.69, $\left.\mathrm{CHCl}_{3}\right) ;{ }^{1} \mathrm{H}$ NMR $\left(400 \mathrm{MHz}, \mathrm{CDCl}_{3}\right): \delta 9.74(1 \mathrm{H}, \mathrm{d}, J=3.1 \mathrm{~Hz}), 7.41-7.21(5 \mathrm{H}, \mathrm{m}), 6.50$ 
$(1 \mathrm{H}, \mathrm{d}, J=15.9 \mathrm{~Hz}), 6.23(1 \mathrm{H}, \mathrm{ddd}, J=15.9,7.0,7.0 \mathrm{~Hz}), 4.54-4.47(1 \mathrm{H}, \mathrm{m}), 4.38(1 \mathrm{H}, \mathrm{dd}, J=$ 7.1, 3.1 Hz), 2.61-2.43 (2H, m), $1.65(3 \mathrm{H}, \mathrm{s}), 1.46(3 \mathrm{H}, \mathrm{s}) ;{ }^{13} \mathrm{C} \mathrm{NMR}\left(100 \mathrm{MHz}, \mathrm{CDCl}_{3}\right): \delta 201.9$ $(+), 137.0(-), 133.3(+), 128.5(2 \mathrm{C},+), 127.4(+), 126.1(2 \mathrm{C},+), 124.7(+), 110.7(-), 81.9(+)$, $78.3(+), 33.4(-), 27.4(+), 25.2(+)$; HRMS (TOF MS ES ${ }^{+}$) calcd for $\mathrm{C}_{15} \mathrm{H}_{18} \mathrm{O}_{3} \mathrm{Na}[\mathrm{M}+\mathrm{Na}]^{+} \mathrm{m} / \mathrm{z}$ 269.1148 , found 269.1166 .

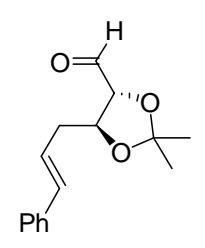

(4R,5S)-2,2-Dimethyl-5-[(E)-3-phenylallyl]-1,3-dioxolane-4-carbaldehyde

To a solution of 3 (1.38 g, $5.60 \mathrm{mmol})$ in $\mathrm{MeOH}(12 \mathrm{~mL})$ at $\mathrm{rt}$ under Ar was added $\mathrm{K}_{2} \mathrm{CO}_{3}(719 \mathrm{mg}, 5.20 \mathrm{mmol})$ and the resulting mixture was stirred for $4 \mathrm{~h}$ at $\mathrm{rt}$. The reaction mixture was then poured into brine $(70 \mathrm{~mL})$ and water $(70 \mathrm{~mL})$ and $\mathrm{CH}_{2} \mathrm{Cl}_{2}$ $(70 \mathrm{~mL})$ were added. The organic phase was isolated and the aqueous phase was extracted with $\mathrm{CH}_{2} \mathrm{Cl}_{2}(2 \times 70 \mathrm{~mL})$. The combined organic phases were dried over $\mathrm{MgSO}_{4}$, filtered, concentrated and dried in vacuo. Flash chromatography of the residue yielded $4(1.15 \mathrm{~g}, 83 \%)$ as a pale yellowish oil: $R_{f}\left(\right.$ cyclohexane/EtOAc 75:25) $=0.24 ;[\alpha]^{23}{ }_{\mathrm{D}}=+15.6\left(c 0.75, \mathrm{CHCl}_{3}\right) ;{ }^{1} \mathrm{H} \mathrm{NMR}$ (400 MHz, $\left.\mathrm{CDCl}_{3}\right): \delta 9.78(1 \mathrm{H}, \mathrm{d}, J=2.0 \mathrm{~Hz}), 7.41-7.21(5 \mathrm{H}, \mathrm{m}), 6.54(1 \mathrm{H}, \mathrm{d}, J=15.9 \mathrm{~Hz}), 6.25$ $(1 \mathrm{H}, \mathrm{ddd}, J=15.9,7.2,7.2 \mathrm{~Hz}), 4.26-4.20(1 \mathrm{H}, \mathrm{m}), 4.11(1 \mathrm{H}, \mathrm{dd}, J=7.5,2.0 \mathrm{~Hz}), 2.74-2.57(2 \mathrm{H}$, m), $1.53(3 \mathrm{H}, \mathrm{s}), 1.46(3 \mathrm{H}, \mathrm{s}) ;{ }^{13} \mathrm{C}$ NMR $\left(100 \mathrm{MHz}, \mathrm{CDCl}_{3}\right): \delta 201.0(+), 137.0(-), 133.5(+)$, $128.5(2 \mathrm{C},+), 127.4(+), 126.2(2 \mathrm{C},+), 124.0(+), 111.1(-), 83.9(+), 76.4(+), 36.4(-), 27.0\left(^{+}\right)$, $26.1(+)$; HRMS (TOF MS ES ${ }^{+}$) calcd for $\mathrm{C}_{15} \mathrm{H}_{18} \mathrm{O}_{3} \mathrm{Na}[\mathrm{M}+\mathrm{Na}]^{+} m / z$ 269.1148, found 269.1154.

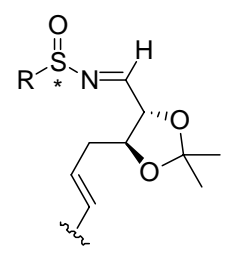

General procedure for synthesis of sulfinimines from aldehydes. To a solution of the aldehyde $(3.60 \mathrm{mmol})$ in $\mathrm{CH}_{2} \mathrm{Cl}_{2}(90 \mathrm{~mL})$ at $\mathrm{rt}$ under $\mathrm{Ar}$ was added the sulfinamide $(3.96 \mathrm{mmol})$ and $\mathrm{Ti}(\mathrm{OEt})_{4}(3.80 \mathrm{~mL}, 18.1 \mathrm{mmol})$ and stirring was continued for $4.5-6 \mathrm{~h}$ at $\mathrm{rt}$. The mixture was cooled to $0{ }^{\circ} \mathrm{C}$ and ice-cold water $(20$ $\mathrm{mL}$ ) was added. The mixture was then filtered through a celite pad, washing the pad with EtOAc $(3 \times 50 \mathrm{~mL})$. The filtrate was washed with brine $(100 \mathrm{~mL})$, dried over $\mathrm{MgSO}_{4}$, filtered, concentrated and dried in vacuo. Flash chromatography of the residue yielded the desired products. 


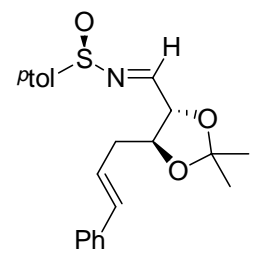

(S)-4-Methylbenzenesulfinic

acid

1-\{(4S,5S)-2,2-dimethyl-5-[(E)-3phenylallyl]-1,3-dioxolan-4-yl\}methylideneamide (5a). Reaction of 4 (444 mg, $1.80 \mathrm{mmol})$ with $(R)-(-)-p$-toluenesulfinamide $(308 \mathrm{mg}, 1.98 \mathrm{mmol})$ for $5 \mathrm{~h}$ following the general procedure yielded 5 a $(344 \mathrm{mg}, 50 \%)$ as a pale yellowish solid: $R_{f}($ cyclohexane $/$ EtOAc $90: 10)=0.28 ; \mathrm{mp}=44-47{ }^{\circ} \mathrm{C} ;[\alpha]^{22}{ }_{\mathrm{D}}=-204.6\left(c 0.55, \mathrm{CHCl}_{3}\right) ;{ }^{1} \mathrm{H}$ NMR (400 MHz, $\left.\mathrm{CDCl}_{3}\right): \delta 8.26(1 \mathrm{H}, \mathrm{d}, J=4.8 \mathrm{~Hz}), 7.59-7.54(2 \mathrm{H}, \mathrm{m}), 7.39-7.21(7 \mathrm{H}, \mathrm{m}), 6.51$ $(1 \mathrm{H}, \mathrm{d}, J=15.9 \mathrm{~Hz}), 6.25(1 \mathrm{H}, \mathrm{ddd}, J=15.9,7.1,7.1 \mathrm{~Hz}), 4.47(1 \mathrm{H}, \mathrm{dd}, J=7.8,4.8 \mathrm{~Hz}), 4.27-$ $4.20(1 \mathrm{H}, \mathrm{m}), 2.74-2.56(2 \mathrm{H}, \mathrm{m}), 2.42(3 \mathrm{H}, \mathrm{s}), 1.50(3 \mathrm{H}, \mathrm{s}), 1.44(3 \mathrm{H}, \mathrm{s}) ;{ }^{13} \mathrm{C}$ NMR $(100 \mathrm{MHz}$, $\left.\mathrm{CDCl}_{3}\right): \delta 164.3(+), 141.9(-), 140.7(-), 137.0(-), 133.3(+), 129.8(2 \mathrm{C},+), 128.4(2 \mathrm{C},+)$, $127.3(+), 126.1(2 \mathrm{C},+), 124.5(2 \mathrm{C},+), 124.3(+), 110.7(-), 80.9(+), 78.2(+), 35.8(-), 27.0(+)$, $26.4(+), 21.4(+)$; HRMS (TOF MS ES ${ }^{+}$) calcd for $\mathrm{C}_{22} \mathrm{H}_{26} \mathrm{NO}_{3} \mathrm{~S}[\mathrm{M}+\mathrm{H}]^{+} m / z$ 384.1628, found 384.1626.

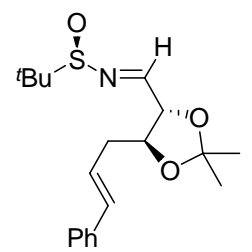

(R)-2-Methylpropane-2-sulfinic acid 1-\{(4S,5S)-2,2-dimethyl-5-[(E)-3phenylallyl]-1,3-dioxolan-4-yl\}methylideneamide (5b). Reaction of 4 (887 mg, $3.60 \mathrm{mmol})$ with $(R)-(+)$-tert-butanesulfinamide $(480 \mathrm{mg}, 3.96 \mathrm{mmol})$ for $6 \mathrm{~h}$ following the general procedure yielded $\mathbf{5 b}(930 \mathrm{mg}, 74 \%)$ as a pale yellowish oil: $R_{f}\left(\right.$ cyclohexane/EtOAc 85:15) $=0.26 ;[\alpha]^{22}{ }_{\mathrm{D}}=-215.5\left(c 0.49, \mathrm{CHCl}_{3}\right) ;{ }^{1} \mathrm{H} \mathrm{NMR}(400 \mathrm{MHz}$, $\left.\mathrm{CDCl}_{3}\right): \delta 8.07(1 \mathrm{H}, \mathrm{d}, J=4.7 \mathrm{~Hz}), 7.37-7.18(5 \mathrm{H}, \mathrm{m}), 6.50(1 \mathrm{H}, \mathrm{d}, J=15.9 \mathrm{~Hz}), 6.25(1 \mathrm{H}, \mathrm{ddd}, J$ $=15.9,7.1,7.1 \mathrm{~Hz}), 4.48(1 \mathrm{H}, \mathrm{dd}, J=7.9,4.7 \mathrm{~Hz}), 4.22-4.16(1 \mathrm{H}, \mathrm{m}), 2.72-2.56(2 \mathrm{H}, \mathrm{m}), 1.50$ $(3 \mathrm{H}, \mathrm{s}), 1.44(3 \mathrm{H}, \mathrm{s}), 1.19(9 \mathrm{H}, \mathrm{s}) ;{ }^{13} \mathrm{C}$ NMR (100 MHz, $\left.\mathrm{CDCl}_{3}\right): \delta 166.8(+), 137.0(-), 133.3$ $(+), 128.4(2 \mathrm{C},+), 127.3(+), 126.1(2 \mathrm{C},+), 124.4(+), 110.7(-), 81.1(+), 78.3(+), 57.2(-), 35.8$ $(-), 27.1(+), 26.4(+), 22.4(3 \mathrm{C},+)$; HRMS (TOF MS ES ${ }^{+}$) calcd for $\mathrm{C}_{19} \mathrm{H}_{28} \mathrm{NO}_{3} \mathrm{~S}[\mathrm{M}+\mathrm{H}]^{+} \mathrm{m} / \mathrm{z}$ 350.1784 , found 350.1798 .

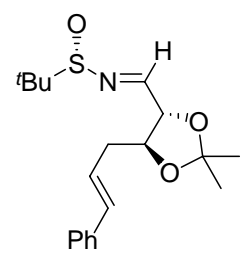

(S)-2-Methylpropane-2-sulfinic acid 1-\{(4S,5S)-2,2-dimethyl-5-[(E)-3phenylallyl]-1,3-dioxolan-4-yl\}methylideneamide (5c). Reaction of 4 (228 mg, $1.17 \mathrm{mmol})$ with $(S)-(-)$-tert-butanesulfinamide $(156 \mathrm{mg}, 1.29 \mathrm{mmol})$ for $5.5 \mathrm{~h}$ following the general procedure yielded 5c (332 mg, as a inseparable mixture with $15 \%$-wt. 4 as judged by NMR; thus the effective calculated yield was $282 \mathrm{mg}, 69 \%$ ) as a pale yellowish oil. An analytical sample of 5c was obtained by subjecting a sample of this 
mixture to the reaction conditions again: $R_{f}\left(\right.$ cyclohexane/EtOAc 80:20) $=0.43 ;[\alpha]^{22}{ }_{\mathrm{D}}=+71.7(c$ 0.70, $\left.\mathrm{CHCl}_{3}\right) ;{ }^{1} \mathrm{H} \mathrm{NMR}\left(400 \mathrm{MHz}, \mathrm{CDCl}_{3}\right): \delta 8.13(1 \mathrm{H}, \mathrm{d}, J=4.6 \mathrm{~Hz}), 7.41-7.21(5 \mathrm{H}, \mathrm{m}), 6.52$ $(1 \mathrm{H}, \mathrm{d}, J=15.9 \mathrm{~Hz}), 6.27(1 \mathrm{H}, \mathrm{ddd}, J=15.9,7.3,7.3 \mathrm{~Hz}), 4.54(1 \mathrm{H}, \mathrm{dd}, J=7.6,4.6 \mathrm{~Hz}), 4.22-$ $4.15(1 \mathrm{H}, \mathrm{m}), 2.74-2.57(2 \mathrm{H}, \mathrm{m}), 1.53(3 \mathrm{H}, \mathrm{s}), 1.46(3 \mathrm{H}, \mathrm{s}), 1.21(9 \mathrm{H}, \mathrm{s}) ;{ }^{13} \mathrm{C}$ NMR $(100 \mathrm{MHz}$, $\left.\mathrm{CDCl}_{3}\right): \delta 167.6(+), 137.0(-), 133.3(+), 128.5(2 \mathrm{C},+), 127.4(+), 126.2(2 \mathrm{C},+), 124.5(+)$, $110.7(-), 81.1(+), 78.5(+), 57.0(-), 36.0(-), 27.1(+), 26.5(+), 22.3(3 \mathrm{C},+)$; HRMS (TOF MS $\mathrm{ES}^{+}$) calcd for $\mathrm{C}_{19} \mathrm{H}_{27} \mathrm{NO}_{3} \mathrm{SNa}[\mathrm{M}+\mathrm{Na}]^{+} \mathrm{m} / z$ 372.1604, found 372.1619 .

$\mathrm{MeO}_{Y} \mathrm{O}$ tert-Butoxycarbonyloxyacetic acid methyl ester (6 Me/Boc). To a solution of methyl Boco glycolate $(1.80 \mathrm{~g}, 20.0 \mathrm{mmol})$ in $\mathrm{CH}_{2} \mathrm{Cl}_{2}(100 \mathrm{~mL})$ at $0{ }^{\circ} \mathrm{C}$ under $\mathrm{Ar}$ was added DMAP (244 mg, $2.00 \mathrm{mmol})$ and $\mathrm{Boc}_{2} \mathrm{O}(4.30 \mathrm{~g}, 19.7 \mathrm{mmol})$. The mixture was then stirred at $0{ }^{\circ} \mathrm{C}$ for 3 $\mathrm{h}$ and at $\mathrm{rt}$ for $1 \mathrm{~h}$. The mixture was concentrated and dried in vacuo and flash chromatography of the residue yielded $6 \mathrm{Me} /$ Boc $(3.18 \mathrm{~g}, 84 \%)$ as a colorless oil which was stored over $4 \AA$ MS: $R_{f}$ $\left(\right.$ cyclohexane/EtOAc 90:10) $=0.29 ;{ }^{1} \mathrm{H}$ NMR $\left(400 \mathrm{MHz}, \mathrm{CDCl}_{3}\right): \delta 4.56(2 \mathrm{H}, \mathrm{s}), 3.77(3 \mathrm{H}, \mathrm{s})$, $1.49(9 \mathrm{H}, \mathrm{s}) ;{ }^{13} \mathrm{C} \mathrm{NMR}\left(100.6 \mathrm{MHz}, \mathrm{CDCl}_{3}\right): \delta 168.2(-), 152.9(-), 83.2(-), 62.6(-), 52.2(+)$, $27.6(3 \mathrm{C},+) .6 \mathrm{Me} /$ Boc is a known compound. ${ }^{\text {S5 }}$

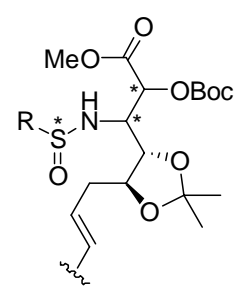

General procedure for reaction of sulfinimines with protected $\alpha$-hydroxyacetates 6. To a solution of $6(10.0 \mathrm{mmol})$ in $\mathrm{THF}(30 \mathrm{~mL})$ at $-78{ }^{\circ} \mathrm{C}$ under $\mathrm{Ar}$ was added $1 \mathrm{M}$ LiHMDS in THF $(10.0 \mathrm{~mL}, 10.0 \mathrm{mmol})$ and the resulting mixture was stirred for $1 \mathrm{~h}$. A solution of the imine $(2.5 \mathrm{mmol})$ in THF $(7.5 \mathrm{~mL})$ was then added dropwise and the resulting mixture was stirred for $0.5-1 \mathrm{~h}$ at $-78^{\circ} \mathrm{C}$. Satd. aq. $\mathrm{NH}_{4} \mathrm{Cl}(75 \mathrm{~mL})$ was added and the mixture was diluted with water $(75 \mathrm{~mL})$. The resulting mixture was extracted with EtOAc $(2 \times 150 \mathrm{~mL})$ and the combined organic phases were dried over $\mathrm{MgSO}_{4}$, filtered, concentrated and dried in vacuo. Flash chromatography of the residue yielded the desired products. 


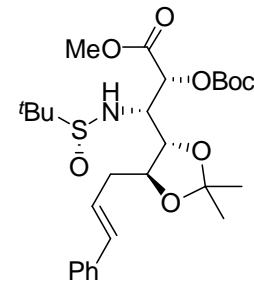

$\left(\right.$ cyclohexane/EtOAc 70:30) $=0.25 ; \mathrm{mp}=110-113{ }^{\circ} \mathrm{C} ;[\alpha]^{21}{ }_{\mathrm{D}}=-37.3\left(c 0.81, \mathrm{CHCl}_{3}\right) ;{ }^{1} \mathrm{H} \mathrm{NMR}$ $\left(400 \mathrm{MHz}, \mathrm{CDCl}_{3}\right): \delta$ 7.40-7.35 (2H, m), 7.31-7.26 (2H, m), 7.23-7.17 $(1 \mathrm{H}, \mathrm{m}), 6.52(1 \mathrm{H}, \mathrm{d}, J=$ $15.9 \mathrm{~Hz}), 6.25(1 \mathrm{H}, \mathrm{ddd}, J=15.9,7.7,6.7 \mathrm{~Hz}), 4.99(1 \mathrm{H}, \mathrm{d}, J=4.0 \mathrm{~Hz}), 4.38(1 \mathrm{H}, \mathrm{ddd}, J=8.6$, 6.1, $6.1 \mathrm{~Hz}), 4.27(1 \mathrm{H}, \mathrm{d}, J=9.0 \mathrm{~Hz}), 3.88(2 \mathrm{H}, \mathrm{m}), 3.63(3 \mathrm{H}, \mathrm{s}), 2.70-2.61(1 \mathrm{H}, \mathrm{m}), 2.58-2.49$ $(1 \mathrm{H}, \mathrm{m}), 1.48(9 \mathrm{H}, \mathrm{s}), 1.42(3 \mathrm{H}, \mathrm{s}), 1.38(3 \mathrm{H}, \mathrm{s}), 1.21(9 \mathrm{H}, \mathrm{s}) ;{ }^{13} \mathrm{C} \mathrm{NMR}\left(100 \mathrm{MHz}, \mathrm{CDCl}_{3}\right): \delta$ $168.2(-), 152.6(-), 137.2(-), 133.3(+), 128.4(2 \mathrm{C},+), 127.2(+), 126.3(2 \mathrm{C},+), 124.7(+)$, $109.2(-), 83.4(-), 79.0(+), 76.2(+), 75.7(+), 56.5(-), 56.2(+), 52.3(+), 35.8(-), 27.7(3 \mathrm{C},+)$, $27.4(+), 26.9(+), 22.6(3 \mathrm{C},+)$; HRMS (TOF MS ES ${ }^{+}$) calcd for $\mathrm{C}_{27} \mathrm{H}_{42} \mathrm{NO}_{8} \mathrm{~S}[\mathrm{M}+\mathrm{H}]^{+} \mathrm{m} / \mathrm{z}$ 540.2626 , found 540.2614 .

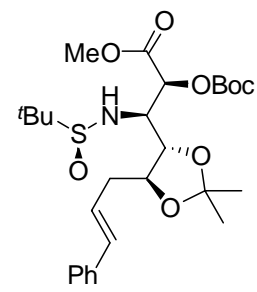

(2S,3R)-2-(tert-Butoxycarbonyloxy)-3-\{(4S,5S)-2,2-dimethyl-5-[(E)-3phenylallyl]-1,3-dioxolan-4-yl\}-3-[(S)-2-methylpropane-2-sulfinylamino]propanoic acid methyl ester (7c). Reaction of sulfinimine 5c (253 $\mathrm{mg}$ of a mixture of $85 \%$-wt 5c and 15\%-wt 4 as judged by NMR; thus effectively 215 $\mathrm{mg}, 0.61 \mathrm{mmol} 5 \mathbf{c})$ with $\mathbf{6} \mathbf{M e} / \mathbf{B o c}(550 \mathrm{mg}, 2.89 \mathrm{mmol})$ for $1 \mathrm{~h}$ following the general procedure yielded 7c (312 mg, 94\%) as a colorless solid: $R_{f}$ (cyclohexane/EtOAc 70:30) $=0.33 ; \mathrm{mp}=161-162{ }^{\circ} \mathrm{C} ;[\alpha]^{21} \mathrm{D}=+33.7\left(c 0.90, \mathrm{CHCl}_{3}\right) ;{ }^{1} \mathrm{H} \mathrm{NMR}\left(400 \mathrm{MHz}, \mathrm{CDCl}_{3}\right): \delta 7.40-$ $7.36(2 \mathrm{H}, \mathrm{m}), 7.30-7.24(2 \mathrm{H}, \mathrm{m}), 7.21-7.15(1 \mathrm{H}, \mathrm{m}), 6.50(1 \mathrm{H}, \mathrm{d}, J=15.9 \mathrm{~Hz}), 6.25(1 \mathrm{H}, \mathrm{ddd}, J=$ $15.9,7.1,6.1 \mathrm{~Hz}), 5.32(1 \mathrm{H}, \mathrm{d}, J=1.9 \mathrm{~Hz}), 4.50(1 \mathrm{H}, \mathrm{ddd}, J=6.6,5.4,5.4 \mathrm{~Hz}), 3.95(1 \mathrm{H}, \mathrm{ddd}, J$ $=10.1,8.4,1.9 \mathrm{~Hz}), 3.86(1 \mathrm{H}, \mathrm{dd}, J=8.4,5.4 \mathrm{~Hz}), 3.72(3 \mathrm{H}, \mathrm{s}), 3.56(1 \mathrm{H}, \mathrm{d}, J=10.1 \mathrm{~Hz}), 2.74-$ $2.65(1 \mathrm{H}, \mathrm{m}), 2.64-2.54(1 \mathrm{H}, \mathrm{m}), 1.50(9 \mathrm{H}, \mathrm{s}), 1.43(6 \mathrm{H}, \mathrm{s}), 1.14(9 \mathrm{H}, \mathrm{s}) ;{ }^{13} \mathrm{C} \mathrm{NMR}(100 \mathrm{MHz}$, $\left.\mathrm{CDCl}_{3}\right): \delta 168.7(-), 152.3(-), 137.4(-), 132.7(+), 128.3(2 \mathrm{C},+), 127.0(+), 126.2(2 \mathrm{C},+)$, $125.9(+), 109.9(-), 83.4(-), 79.8(+), 79.1\left(^{+}\right), 74.4(+), 60.9(+), 56.7(-), 52.3(+), 38.1\left(^{(}\right)$, $27.8(+), 27.7(4 \mathrm{C},+), 22.5(3 \mathrm{C},+)$; HRMS (TOF MS ES ${ }^{+}$) calcd for $\mathrm{C}_{27} \mathrm{H}_{42} \mathrm{NO}_{8} \mathrm{~S}[\mathrm{M}+\mathrm{H}]^{+} \mathrm{m} / \mathrm{z}$ 540.2626 , found 540.2625 . 


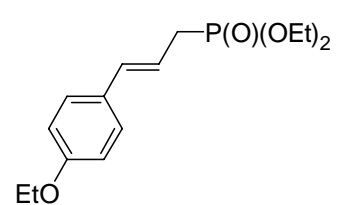

(E)-3-[(4-Ethoxyphenyl)allyl]phosphonic acid diethyl ester (S1). To a mixture of 4-iodophenetole $(2.49 \mathrm{~g}, 10.0 \mathrm{mmol})$ in $\mathrm{Et}_{3} \mathrm{~N}(2.10 \mathrm{~mL}, 15.1$ $\mathrm{mmol})$ at $\mathrm{rt}$ under Ar was added diethyl allylphosphonate $(1.75 \mathrm{~mL}, 10.0$ $\mathrm{mmol}), \mathrm{Pd}(\mathrm{OAc})_{2}(45 \mathrm{mg}, 0.20 \mathrm{mmol})$ and $\mathrm{PPh}_{3}(105 \mathrm{mg}$. $0.40 \mathrm{mmol})$. After stirring for $2.5 \mathrm{~h}$ at $100{ }^{\circ} \mathrm{C}$, further 4-iodophenetole (495 mg, $2.00 \mathrm{mmol}$ ) was added and stirring was continued for $1.5 \mathrm{~h}$ further at $100{ }^{\circ} \mathrm{C}$. The mixture was cooled to $\mathrm{rt}$ and $\mathrm{Et}_{2} \mathrm{O}(25 \mathrm{~mL})$ and water $(10 \mathrm{~mL})$ were added. The organic phase was isolated and the aqueous phase was extracted with $\mathrm{Et}_{2} \mathrm{O}(4 \times 25$ $\mathrm{mL}$ ). The combined organic phases were dried over $\mathrm{MgSO}_{4}$, filtered and concentrated under reduced pressure. Flash chromatography of the residue yielded $\mathrm{S1}(1.52 \mathrm{~g}, 51 \%)$ as a yellowish solid: $R_{f}$ (cyclohexane/EtOAc 25:75) $=0.35 ; \mathrm{mp}=31-33{ }^{\circ} \mathrm{C} ;{ }^{1} \mathrm{H}$ NMR $\left(400 \mathrm{MHz}, \mathrm{CDCl}_{3}\right): \delta 7.27$ $(2 \mathrm{H}, \mathrm{d}, J=8.8 \mathrm{~Hz}), 6.82(2 \mathrm{H}, \mathrm{d}, J=8.8 \mathrm{~Hz}), 6.54(1 \mathrm{H}, \mathrm{dd}, J=15.8,5.2 \mathrm{~Hz}), 6.06-5.95(1 \mathrm{H}, \mathrm{m})$, 4.16-4.05 (4H, m), $4.02(2 \mathrm{H}, \mathrm{q}, J=7.0 \mathrm{~Hz}), 2.73(2 \mathrm{H}, \mathrm{ddd}, J=22.0,7.6,1.0 \mathrm{~Hz}), 1.40(3 \mathrm{H}, \mathrm{t}, J=$ $7.0 \mathrm{~Hz}), 1.31(6 \mathrm{H}, \mathrm{t}, J=7.1 \mathrm{~Hz}) ;{ }^{13} \mathrm{C} \mathrm{NMR}\left(100 \mathrm{MHz}, \mathrm{CDCl}_{3}\right): \delta 158.4(-), 134.0(+, \mathrm{d}, J=15.0$ Hz), $129.4(-, \mathrm{d}, J=2.9 \mathrm{~Hz}), 127.2(2 \mathrm{C},+), 116.1(+, \mathrm{d}, J=12.0 \mathrm{~Hz}), 114.4(2 \mathrm{C},+), 63.3(-)$, $61.9(2 \mathrm{C},-, \mathrm{d}, J=6.7 \mathrm{~Hz}), 30.9$ (-, d, $J=140 \mathrm{~Hz}), 16.4(2 \mathrm{C},+$, d, $J=5.6 \mathrm{~Hz}), 14.7$ (+); HRMS $\left(\right.$ TOF MS ES ${ }^{+}$) calcd for $\mathrm{C}_{15} \mathrm{H}_{24} \mathrm{O}_{4} \mathrm{P}[\mathrm{M}+\mathrm{H}]^{+} m / z$ 299.1407, found 299.1415.

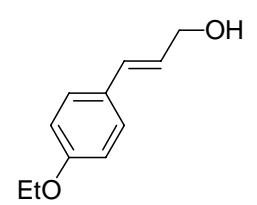

(E)-3-(4-Ethoxyphenyl)prop-2-enol (S2). To a solution of ethyl trans-4-ethoxycinnamate $(2.00 \mathrm{~g}, 8.80 \mathrm{mmol})$ in $\mathrm{CH}_{2} \mathrm{Cl}_{2}(35 \mathrm{~mL})$ at $-78^{\circ} \mathrm{C}$ under $\mathrm{Ar}$ was added dropwise 1.0M DIBAL-H in $\mathrm{CH}_{2} \mathrm{Cl}_{2}(20.0 \mathrm{~mL}, 20.0 \mathrm{mmol})$. The mixture was warmed to $-40^{\circ} \mathrm{C}$ and stirring was continued for $2 \mathrm{~h}$ further. The mixture was diluted with $\mathrm{Et}_{2} \mathrm{O}$ $(45 \mathrm{~mL})$ and was poured into $1 \mathrm{M}$ aq. Rochelle's salt $(35 \mathrm{~mL})$. The resulting mixture was stirred vigorously at $\mathrm{rt}$ for $19 \mathrm{~h}$. The organic phase was isolated and the aqueous phase was extracted with $\mathrm{Et}_{2} \mathrm{O}(2 \times 70 \mathrm{~mL})$. The combined organic layers were dried over $\mathrm{MgSO}_{4}$, filtered and concentrated under reduced pressure. Flash chromatography of the residue yielded S2 $(1.57 \mathrm{~g}$, $100 \%$ ) as a colorless solid: $R_{f}$ (cyclohexane/EtOAc 60:40) $=0.44 ; \mathrm{mp}=89-91{ }^{\circ} \mathrm{C}\left(\right.$ lit. $^{\mathrm{S} 6} \mathrm{mp}=90$ $\left.91{ }^{\circ} \mathrm{C}\right) ;{ }^{1} \mathrm{H}$ NMR (400 MHz, $\left.\mathrm{CDCl}_{3}\right): \delta 7.31(2 \mathrm{H}, \mathrm{d}, J=8.7 \mathrm{~Hz}), 6.85(2 \mathrm{H}, \mathrm{d}, J=8.7 \mathrm{~Hz}), 6.55$ $(1 \mathrm{H}, \mathrm{d}, J=15.9 \mathrm{~Hz}), 6.06-5.95(1 \mathrm{H}, \mathrm{dt}, J=15.9,5.7 \mathrm{~Hz}), 4.29(1 \mathrm{H}, \mathrm{d}, J=5.7 \mathrm{~Hz}), 4.03(2 \mathrm{H}, \mathrm{q}, J$ $=7.0 \mathrm{~Hz}), 1.56-1.50\left(1 \mathrm{H}\right.$, br s), $1.41(3 \mathrm{H}, \mathrm{t}, J=7.0 \mathrm{~Hz}) ;{ }^{13} \mathrm{C} \mathrm{NMR}\left(100 \mathrm{MHz}, \mathrm{CDCl}_{3}\right): \delta 158.7$ $(-), 131.0(+), 129.2(-), 127.6(2 \mathrm{C},+), 126.1(+), 114.6(2 \mathrm{C},+), 63.9(-), 63.4(-), 14.8(+)$; 
HRMS (TOF MS ES ${ }^{+}$) calcd for $\mathrm{C}_{11} \mathrm{H}_{13} \mathrm{O}\left[\mathrm{M}-\mathrm{H}_{2} \mathrm{O}+\mathrm{H}\right]^{+} \mathrm{m} / z$ 161.0961, found 161.0959. S2 is a known compound. ${ }^{\mathrm{S} 6, \mathrm{~S} 7}$

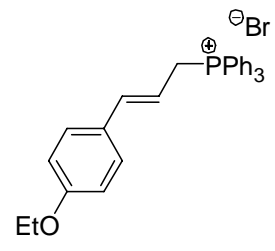

$\{(E)-3-[(4-E t h o x y p h e n y l) a l l y l]\}$ triphenylphosphonium bromide (8). To a solution of S2 (1.39 g, $7.80 \mathrm{mmol})$ in THF $(115 \mathrm{~mL})$ at $\mathrm{rt}$ under Ar was added $\mathrm{PPh}_{3}(5.12 \mathrm{~g}, 19.5 \mathrm{mmol})$ and $N$-bromosuccinimide $(1.67 \mathrm{~g}, 9.38 \mathrm{mmol})$ and the resulting mixture was refluxed in the dark for $18 \mathrm{~h}$. The mixture was allowed to cool to $\mathrm{rt}$ and the formed precipitates were filtered off, washed with a little ice-cold THF, and dried in vacuo, yielding 8 as a THF complex $(2.90 \mathrm{~g}, \mathbf{8} / \mathrm{THF}=1.0: 0.6,68 \%)$ as a pale tan solid which was used in the ensuing reaction without further purification: $\mathrm{mp}=160-163{ }^{\circ} \mathrm{C}$; ${ }^{1} \mathrm{H}$ NMR (400 MHz, $\left.\mathrm{CDCl}_{3}\right): \delta$ 7.87-7.79 (6H, m), 7.79-7.72 (3H, m), 7.69-7.61 (6H, m), 7.09 $(2 \mathrm{H}, \mathrm{d}, J=8.6 \mathrm{~Hz}), 6.73(2 \mathrm{H}, \mathrm{d}, J=8.6 \mathrm{~Hz}), 6.68(1 \mathrm{H}, \mathrm{dd}, J=15.8,5.6 \mathrm{~Hz}), 5.84-5.73(1 \mathrm{H}, \mathrm{m})$, $4.89(1 \mathrm{H}, \mathrm{dd}, J=15.1,7.5 \mathrm{~Hz}), 3.96(2 \mathrm{H}, \mathrm{q}, J=7.0 \mathrm{~Hz}), 3.76-3.70(2.4 \mathrm{H}, \mathrm{m}), 1.87-1.81(2.4 \mathrm{H}$, m), $1.35(3 \mathrm{H}, \mathrm{t}, J=7.0 \mathrm{~Hz}) ;{ }^{13} \mathrm{C} \mathrm{NMR}\left(100 \mathrm{MHz}, \mathrm{CDCl}_{3}\right): \delta 159.1(-), 139.7(+, \mathrm{d}, J=13.5 \mathrm{~Hz})$, $134.9(3 \mathrm{C},+, \mathrm{d}, J=2.1 \mathrm{~Hz}), 134.0(6 \mathrm{C},+, \mathrm{d}, J=9.7 \mathrm{~Hz}), 130.2(6 \mathrm{C},+, \mathrm{d}, J=12.4 \mathrm{~Hz}), 128.3$ (-, d, $J=3.6 \mathrm{~Hz}), 127.8(+), 118.1(3 \mathrm{C},-, \mathrm{d}, J=85.4 \mathrm{~Hz}), 114.4(2 \mathrm{C},+), 110.6(+, \mathrm{d}, J=10.9 \mathrm{~Hz})$, $67.9(2 \times 0.6 \mathrm{C},-), 63.4(-), 28.3(-, \mathrm{d}, J=48.8 \mathrm{~Hz}), 25.5(2 \times 0.6 \mathrm{C},-), 14.7(+)$; HRMS (TOF MS $\mathrm{ES}^{+}$) calcd for $\mathrm{C}_{29} \mathrm{H}_{28} \mathrm{OP}[\mathrm{M}]^{+} \mathrm{m} / z$ 423.1872, found 423.1868. NMR spectra were, apart from the THF peaks, in full accordance with those reported in the literature for uncomplexed $8{ }^{\mathrm{S} 8}$

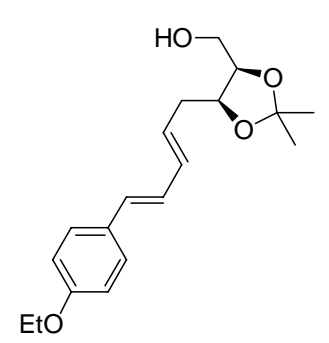

\{(4R,5S)-5-[(2E,4E)-5-(4-Ethoxyphenyl)penta-2,4-dienyl]-2,2-dimethyl1,3-dioxolan-4-yl\}methanol $\quad[(E)-9] \quad$ and $\quad\{(4 R, 5 S)-5-[(2 Z, 4 E)-5-$ (4-ethoxyphenyl)penta-2,4-dienyl]-2,2-dimethyl-1,3-dioxolan4-yl\}methanol [(Z)-9]. To a suspension of 9·0.6THF (1.51 g, $2.76 \mathrm{mmol})$ in benzene $(50 \mathrm{~mL})$ at $0{ }^{\circ} \mathrm{C}$ under Ar was added dropwise 0.5 M KHMDS in toluene $(6.0 \mathrm{~mL}, 3.00 \mathrm{mmol})$. The mixture was allowed to warm to $\mathrm{rt}$ for $10 \mathrm{~min}$ and was then cooled to $0{ }^{\circ} \mathrm{C}$. A solution of $\mathbf{1}^{\mathrm{S} 4}(262 \mathrm{mg}, 1.50 \mathrm{mmol})$ in benzene $(25 \mathrm{~mL})$ was added and the resulting solution was stirred for $1.5 \mathrm{~h}$ at $\mathrm{rt}$. $\mathrm{MeOH}(15 \mathrm{~mL})$ and satd. aq. $\mathrm{NH}_{4} \mathrm{Cl}(150 \mathrm{~mL})$ were added and the mixture was extracted with EtOAc $(3 \times 250 \mathrm{~mL})$. The combined organic phases were dried over $\mathrm{MgSO}_{4}$, filtered, concentrated and dried in vacuo. Flash chromatography of the 
residue yielded $(\boldsymbol{E})-\mathbf{9}(235 \mathrm{mg}, 49 \%)$ as a pale yellowish solid and $(Z)-9(91 \mathrm{mg}, 19 \%)$ as a pale yellowish oil.

(E)-9: $R_{f}\left(\right.$ cyclohexane/EtOAc 60:40) $=0.46 ; \mathrm{mp}=54-57{ }^{\circ} \mathrm{C} ;[\alpha]^{21} \mathrm{D}=-13.2\left(c 0.87, \mathrm{CHCl}_{3}\right) ;{ }^{1} \mathrm{H}$ NMR (400 MHz, $\left.\mathrm{CDCl}_{3}\right): \delta 7.29(2 \mathrm{H}, \mathrm{d}, J=8.7 \mathrm{~Hz}), 6.83(2 \mathrm{H}, \mathrm{d}, J=8.7 \mathrm{~Hz}), 6.62(1 \mathrm{H}, \mathrm{dd}, J=$ 15.6, $10.4 \mathrm{~Hz}), 6.49(1 \mathrm{H}, \mathrm{d}, J=15.6 \mathrm{~Hz}), 6.27(1 \mathrm{H}, \mathrm{dd}, J=15.1,10.4 \mathrm{~Hz}), 5.75(1 \mathrm{H}, \mathrm{ddd}, J=$ 15.1, 7.2, 7.2 Hz), $4.26(1 \mathrm{H}, \mathrm{ddd}, J=7.9,5.7,5.7 \mathrm{~Hz}), 4.19$ (1H, ddd, $J=5.7,5.7,5.7 \mathrm{~Hz}), 4.02$ $(2 \mathrm{H}, \mathrm{q}, J=7.0 \mathrm{~Hz}), 3.70(2 \mathrm{H}, \mathrm{d}, J=5.7 \mathrm{~Hz}), 2.54-2.44(1 \mathrm{H}, \mathrm{m}), 2.40-2.31(1 \mathrm{H}, \mathrm{m}), 2.06-1.84$ $\left(1 \mathrm{H}\right.$, br s), $1.50(3 \mathrm{H}, \mathrm{s}), 1.41(3 \mathrm{H}, \mathrm{t}, J=7.0 \mathrm{~Hz}), 1.38(3 \mathrm{H}, \mathrm{s}) ;{ }^{13} \mathrm{C} \mathrm{NMR}\left(100 \mathrm{MHz}, \mathrm{CDCl}_{3}\right): \delta$ $158.5(-), 133.2(+), 131.0(+), 130.0(-), 128.6(+), 127.4(2 \mathrm{C},+), 126.6(+), 114.6(2 \mathrm{C},+)$, $108.3(-), 77.8(+), 76.6(+), 63.4(-), 61.7(-), 32.8(-), 28.1(+), 25.4(+), 14.8(+)$;HRMS (TOF MS ES ${ }^{+}$) calcd for $\mathrm{C}_{19} \mathrm{H}_{26} \mathrm{O}_{4} \mathrm{Na}[\mathrm{M}+\mathrm{Na}]^{+} m / z$ 341.1723, found 341.1739.

(Z)-9: $R_{f}$ (cyclohexane/EtOAc 60:40) $=0.52 ;[\alpha]^{21}{ }_{\mathrm{D}}=+25.9\left(c \quad 0.56, \mathrm{CHCl}_{3}\right) ;{ }^{1} \mathrm{H}$ NMR $(400$ $\left.\mathrm{MHz}, \mathrm{CDCl}_{3}\right): \delta 7.34(2 \mathrm{H}, \mathrm{d}, J=8.7 \mathrm{~Hz}), 6.88(1 \mathrm{H}, \mathrm{dd}, J=15.6,10.9 \mathrm{~Hz}), 6.85(2 \mathrm{H}, \mathrm{d}, J=8.7$ $\mathrm{Hz}), 6.52(1 \mathrm{H}, \mathrm{d}, J=15.6 \mathrm{~Hz}), 6.25(1 \mathrm{H}, \mathrm{dd}, J=10.9,10.9 \mathrm{~Hz}), 5.46(1 \mathrm{H}, \mathrm{ddd}, J=10.9,7.8,7.8$ $\mathrm{Hz}), 4.27(1 \mathrm{H}, \mathrm{dd}, J=6.0,6.0 \mathrm{~Hz}), 4.19(1 \mathrm{H}, \mathrm{dd}, J=6.0,6.0 \mathrm{~Hz}), 4.04(2 \mathrm{H}, \mathrm{q}, J=6.9 \mathrm{~Hz}), 3.70$ $(2 \mathrm{H}, \mathrm{d}, J=6.0 \mathrm{~Hz}), 2.67-2.50(2 \mathrm{H}, \mathrm{m}), 2.04-1.65(1 \mathrm{H}, \mathrm{br} \mathrm{s}), 1.50(3 \mathrm{H}, \mathrm{s}), 1.41(3 \mathrm{H}, \mathrm{t}, J=6.9 \mathrm{~Hz})$, $1.38(3 \mathrm{H}, \mathrm{s}) ;{ }^{13} \mathrm{C} \mathrm{NMR}\left(100 \mathrm{MHz}, \mathrm{CDCl}_{3}\right): 158.7(-), 133.2(+), 131.2(+), 129.9(-), 127.7(2 \mathrm{C}$, +), $125.6(+), 121.6(+), 114.6(2 \mathrm{C},+), 108.3(-), 77.8(+), 76.6(+), 63.5(-), 61.7(-), 28.2(-)$, $28.1(+), 25.4(+), 14.8(+)$; HRMS (TOF MS ES ${ }^{+}$) calcd for $\mathrm{C}_{19} \mathrm{H}_{26} \mathrm{O}_{4} \mathrm{Na}[\mathrm{M}+\mathrm{Na}]^{+} \mathrm{m} / \mathrm{z}$ 341.1723 , found 341.1736. The products were stored under $\mathrm{Ar}$ in the fridge as solutions in $\mathrm{CHCl}_{3}$ since they were unstable in pure, dry form.

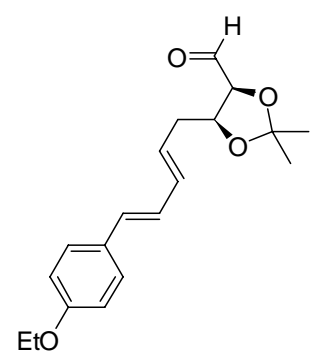

(4S,5S)-5-[(2E,4E)-5-(4-Ethoxyphenyl)penta-2,4-dienyl]-2,2-dimethyl1,3-dioxolane-4-carbaldehyde (10). Reaction of (E)-9 (225 mg, 0.707 $\mathrm{mmol}$ ) following the same procedure as for the conversion of $\mathbf{2}$ to 3 yielded $10(174 \mathrm{mg}, 78 \%)$ as a pale yellowish solid: $R_{f}$ (cyclohexane/EtOAc 75:25) = $0.33 ; \mathrm{mp}=79-81{ }^{\circ} \mathrm{C} ;[\alpha]^{21} \mathrm{D}=-40.8\left(c 0.62, \mathrm{CHCl}_{3}\right) ;{ }^{1} \mathrm{H} \mathrm{NMR}(400 \mathrm{MHz}$, $\left.\mathrm{CDCl}_{3}\right): \delta 9.69(1 \mathrm{H}, \mathrm{d}, J=3.1 \mathrm{~Hz}), 7.30(2 \mathrm{H}, \mathrm{d}, J=8.7 \mathrm{~Hz}), 6.83(2 \mathrm{H}, \mathrm{d}, J=$ $8.7 \mathrm{~Hz}), 6.62(1 \mathrm{H}, \mathrm{dd}, J=15.6,10.3 \mathrm{~Hz}), 6.44(1 \mathrm{H}, \mathrm{d}, J=15.6 \mathrm{~Hz}), 6.25(1 \mathrm{H}, \mathrm{dd}, J=15.1,10.3$ $\mathrm{Hz}), 5.73(1 \mathrm{H}, \mathrm{ddd}, J=15.1,7.3,7.3 \mathrm{~Hz}), 4.47-4.40(1 \mathrm{H}, \mathrm{m}), 4.33(1 \mathrm{H}, \mathrm{dd}, J=7.1,3.1 \mathrm{~Hz}), 4.03$ $(2 \mathrm{H}, \mathrm{q}, J=7.0 \mathrm{~Hz}), 2.49-2.33(2 \mathrm{H}, \mathrm{m}), 1.61(3 \mathrm{H}, \mathrm{s}), 1.42(3 \mathrm{H}, \mathrm{s}), 1.41(3 \mathrm{H}, \mathrm{t}, J=7.0 \mathrm{~Hz}) ;{ }^{13} \mathrm{C}$ 
NMR (100 MHz, $\left.\mathrm{CDCl}_{3}\right): \delta 201.9(+), 158.5(-), 134.1(+), 131.4(+), 129.9(-), 127.5(2 \mathrm{C},+)$, $127.4(+), 126.4(+), 114.6(2 \mathrm{C},+), 110.7(-), 81.9(+), 78.3(+), 63.4(-), 33.2(-), 27.5(+), 25.2$ $(+), 14.8(+)$; HRMS (TOF MS ES ${ }^{+}$) calcd for $\mathrm{C}_{19} \mathrm{H}_{24} \mathrm{O}_{4} \mathrm{Na}[\mathrm{M}+\mathrm{Na}]^{+} \mathrm{m} / z$ 339.1567, found 339.1584. After drying, the product was used immediately in the ensuing reaction.

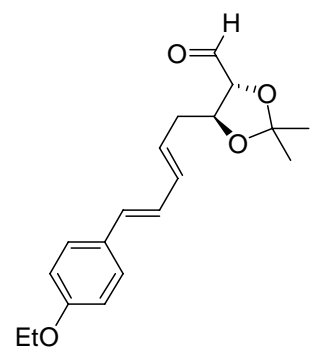

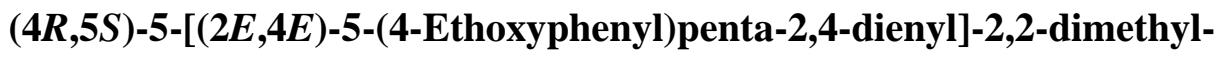
1,3-dioxolane-4-carbaldehyde (11). Reaction of 10 (159 mg, $0.503 \mathrm{mmol})$ in $\mathrm{MeOH}(2.0 \mathrm{~mL})$ for $2.5 \mathrm{~h}$ but otherwise following the same procedure as for the conversion of $\mathbf{3}$ to $\mathbf{4}$ yielded $\mathbf{1 1}$ (123 $\mathrm{mg}, 78 \%$ ) as a pale yellowish oil: $R_{f}\left(\right.$ cyclohexane/EtOAc 70:30) $=0.32 ;[\alpha]^{22}{ }_{\mathrm{D}}=+4.5\left(c 0.62, \mathrm{CHCl}_{3}\right) ;{ }^{1} \mathrm{H}$ NMR (400 MHz, $\left.\mathrm{CDCl}_{3}\right): \delta 9.74(1 \mathrm{H}, \mathrm{d}, J=2.0 \mathrm{~Hz}), 7.30(2 \mathrm{H}, \mathrm{d}, J=8.7$ $\mathrm{Hz}), 6.83(2 \mathrm{H}, \mathrm{d}, J=8.7 \mathrm{~Hz}), 6.63(1 \mathrm{H}, \mathrm{dd}, J=15.6,10.3 \mathrm{~Hz}), 6.44(1 \mathrm{H}, \mathrm{d}, J=15.6 \mathrm{~Hz}), 6.30$ $(1 \mathrm{H}, \mathrm{dd}, J=15.1,10.3 \mathrm{~Hz}), 5.74(1 \mathrm{H}$, ddd, $J=15.1,7.3,7.3 \mathrm{~Hz}), 4.19-4.13(1 \mathrm{H}, \mathrm{m}), 4.06-3.99$ $(1 \mathrm{H}, \mathrm{m}), 4.03(2 \mathrm{H}, \mathrm{q}, J=7.0 \mathrm{~Hz}), 2.63-2.46(2 \mathrm{H}, \mathrm{m}), 1.50(3 \mathrm{H}, \mathrm{s}), 1.43(3 \mathrm{H}, \mathrm{s}), 1.41(3 \mathrm{H}, \mathrm{t}, J=$ $7.0 \mathrm{~Hz}) ;{ }^{13} \mathrm{C} \mathrm{NMR}\left(100 \mathrm{MHz}, \mathrm{CDCl}_{3}\right): \delta 201.0(+), 158.5(-), 134.4(+), 131.4(+), 129.9(-)$, $127.5(2 \mathrm{C},+), 126.8(+), 126.5(+), 114.6(2 \mathrm{C},+), 111.1(-), 84.0(+), 76.5(+), 63.4(-), 36.3(-)$, $27.0(+), 26.2(+), 14.8(+)$; HRMS (TOF MS ES ${ }^{+}$) calcd for $\mathrm{C}_{19} \mathrm{H}_{25} \mathrm{O}_{4}[\mathrm{M}+\mathrm{H}]^{+} \mathrm{m} / z$ 317.1747, found 317.1764. The product was stored overnight under $\mathrm{Ar}$ in the fridge as a solution in $\mathrm{CHCl}_{3}$ since it was unstable in pure, dry form.

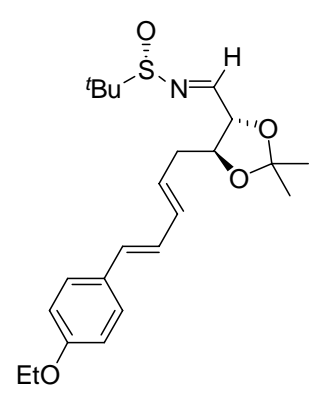

(S)-2-Methylpropane-2-sulfinic acid 1-\{(4S,5S)-5-[(2E,4E)-5-[(4ethoxyphenyl)penta-2,4-dienyl]]-2,2-dimethyl-1,3-dioxolan-4yl\}methylideneamide (12). Reaction of 11 (110 mg, $0.348 \mathrm{mmol})$ with $(S)$ (-)-tert-butanesulfinamide (47 $\mathrm{mg}, 0.388 \mathrm{mmol}$ ) for $4.5 \mathrm{~h}$ with addition of further $(S)$-(-)-tert-butanesulfinamide $(10 \mathrm{mg}, 0.083 \mathrm{mmol})$ after $2 \mathrm{~h}$ but otherwise following the general procedure yielded $12(102 \mathrm{mg}, 70 \%)$ as a colorless solid: $R_{f}$ (cyclohexane/EtOAc 80:20) $=0.45 ; \mathrm{mp}=74-76{ }^{\circ} \mathrm{C} ;[\alpha]^{21} \mathrm{D}=+28.6(c 0.49$, $\left.\mathrm{CHCl}_{3}\right) ;{ }^{1} \mathrm{H}$ NMR $\left(400 \mathrm{MHz}, \mathrm{CDCl}_{3}\right): \delta 8.08(1 \mathrm{H}, \mathrm{d}, J=4.7 \mathrm{~Hz}), 7.29(2 \mathrm{H}, \mathrm{d}, J=8.7 \mathrm{~Hz}), 6.83$ $(2 \mathrm{H}, \mathrm{d}, J=8.7 \mathrm{~Hz}), 6.60(1 \mathrm{H}, \mathrm{dd}, J=15.6,10.3 \mathrm{~Hz}), 6.43(1 \mathrm{H}, \mathrm{d}, J=15.6 \mathrm{~Hz}), 6.27(1 \mathrm{H}, \mathrm{dd}, J=$ 15.1, $10.3 \mathrm{~Hz}), 5.75(1 \mathrm{H}, \mathrm{ddd}, J=15.1,7.3,7.3 \mathrm{~Hz}), 4.47(1 \mathrm{H}, \mathrm{dd}, J=7.6,4.7 \mathrm{~Hz}), 4.14-4.07$ $(1 \mathrm{H}, \mathrm{m}), 4.03(2 \mathrm{H}, \mathrm{q}, J=7.0 \mathrm{~Hz}), 2.63-2.46(2 \mathrm{H}, \mathrm{m}), 1.49(3 \mathrm{H}, \mathrm{s}), 1.43(3 \mathrm{H}, \mathrm{s}), 1.41(3 \mathrm{H}, \mathrm{t}, J=$ 
$7.0 \mathrm{~Hz}), 1.21(9 \mathrm{H}, \mathrm{s}) ;{ }^{13} \mathrm{C} \mathrm{NMR}\left(100 \mathrm{MHz}, \mathrm{CDCl}_{3}\right): \delta 167.6(+), 158.5(-), 134.1(+), 131.3(+)$, $129.9(-), 127.4(2 \mathrm{C},+), 127.2(+), 126.5(+), 114.6(2 \mathrm{C},+), 110.7(-), 81.2(+), 78.6(+), 63.5$ $(-), 57.0(-), 35.9(-), 27.1(+), 26.5(+), 22.4(3 \mathrm{C},+), 14.8(+)$; HRMS (TOF MS ES ${ }^{+}$) calcd for $\mathrm{C}_{23} \mathrm{H}_{33} \mathrm{NO}_{4} \mathrm{SNa}[\mathrm{M}+\mathrm{Na}]^{+} m / z$ 442.2023, found 442.2023. After drying, the product was used immediately in the ensuing reaction.

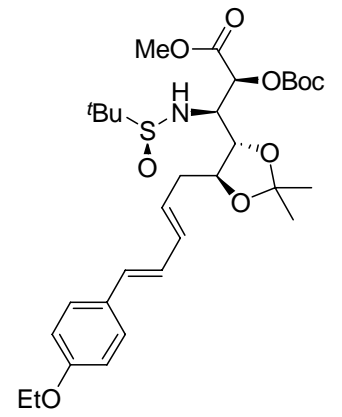

(2S,3R)-2-(tert-Butoxycarbonyloxy)-3-\{(4S,5S)-5-[(2E,4E)-5-(4ethoxyphenyl)penta-2,4-dienyl]-2,2-dimethyl-1,3-dioxolan-4-yl\}-3-[(S)2-methylpropane-2-sulfinylamino]propanoic acid methyl ester (13). Reaction of sulfinimine $12(85 \mathrm{mg}, 0.203 \mathrm{mmol})$ with $6 \mathrm{Me} /$ Boc $(164 \mathrm{mg}$, $0.862 \mathrm{mmol})$ and $1 \mathrm{M}$ LiHMDS in THF $(0.86 \mathrm{~mL}, 0.860 \mathrm{mmol})$ for $1 \mathrm{~h} \mathrm{but}$ otherwise following the general procedure yielded $13(97 \mathrm{mg}, 79 \%)$ as a colorless solid: $R_{f}$ (cyclohexane/EtOAc 70:30) $=0.41 ; \mathrm{mp}=111-112{ }^{\circ} \mathrm{C} ;[\alpha]^{21} \mathrm{D}=+33.6(c 0.50$, $\left.\mathrm{CHCl}_{3}\right) ;{ }^{1} \mathrm{H}$ NMR $\left(400 \mathrm{MHz}, \mathrm{CDCl}_{3}\right): \delta 7.28(2 \mathrm{H}, \mathrm{d}, J=8.6 \mathrm{~Hz}), 6.83(2 \mathrm{H}, \mathrm{d}, J=8.6 \mathrm{~Hz}), 6.64$ $(1 \mathrm{H}, \mathrm{dd}, J=15.6,10.4 \mathrm{~Hz}), 6.42(1 \mathrm{H}, \mathrm{d}, J=15.6 \mathrm{~Hz}), 6.27(1 \mathrm{H}, \mathrm{dd}, J=15.1,10.4 \mathrm{~Hz}), 5.84(1 \mathrm{H}$, ddd, $J=15.1,7.4,7.4 \mathrm{~Hz}), 5.31(1 \mathrm{H}, \mathrm{d}, J=1.5 \mathrm{~Hz}), 4.47-4.40(1 \mathrm{H}, \mathrm{m}), 4.03(2 \mathrm{H}, \mathrm{q}, J=7.0 \mathrm{~Hz})$, 3.97-3.89 (1H, m), $3.82(1 \mathrm{H}, \mathrm{dd}, J=8.5,5.6 \mathrm{~Hz}), 3.73(3 \mathrm{H}, \mathrm{s}), 3.57(1 \mathrm{H}, \mathrm{d}, J=10.1 \mathrm{~Hz}), 2.68-$ $2.59(1 \mathrm{H}, \mathrm{m}), 2.54-2.45(1 \mathrm{H}, \mathrm{m}), 1.51(9 \mathrm{H}, \mathrm{s}), 1.42(6 \mathrm{H}, \mathrm{s}), 1.40(3 \mathrm{H}, \mathrm{t}, J=7.0 \mathrm{~Hz}), 1.17(9 \mathrm{H}, \mathrm{s})$; ${ }^{13} \mathrm{C}$ NMR (100 MHz, $\left.\mathrm{CDCl}_{3}\right): \delta 168.8(-), 158.4(-), 152.4(-), 133.5(+), 130.6(+), 130.2(-)$, $129.0(+), 127.4(2 \mathrm{C},+), 127.1(+), 114.6(2 \mathrm{C},+), 109.9(-), 83.5(-), 79.7(+), 79.3(+), 74.5(+)$, $63.4(-), 60.9(+), 56.8(-), 52.3(+), 38.0(-), 27.8(+), 27.7(4 \mathrm{C},+), 22.6(3 \mathrm{C},+), 14.8(+)$; HRMS (TOF MS ES ${ }^{+}$) calcd for $\mathrm{C}_{31} \mathrm{H}_{47} \mathrm{NO}_{9} \mathrm{SNa}[\mathrm{M}+\mathrm{Na}]^{+} \mathrm{m} / z$ 632.2864, found 628.2868. 

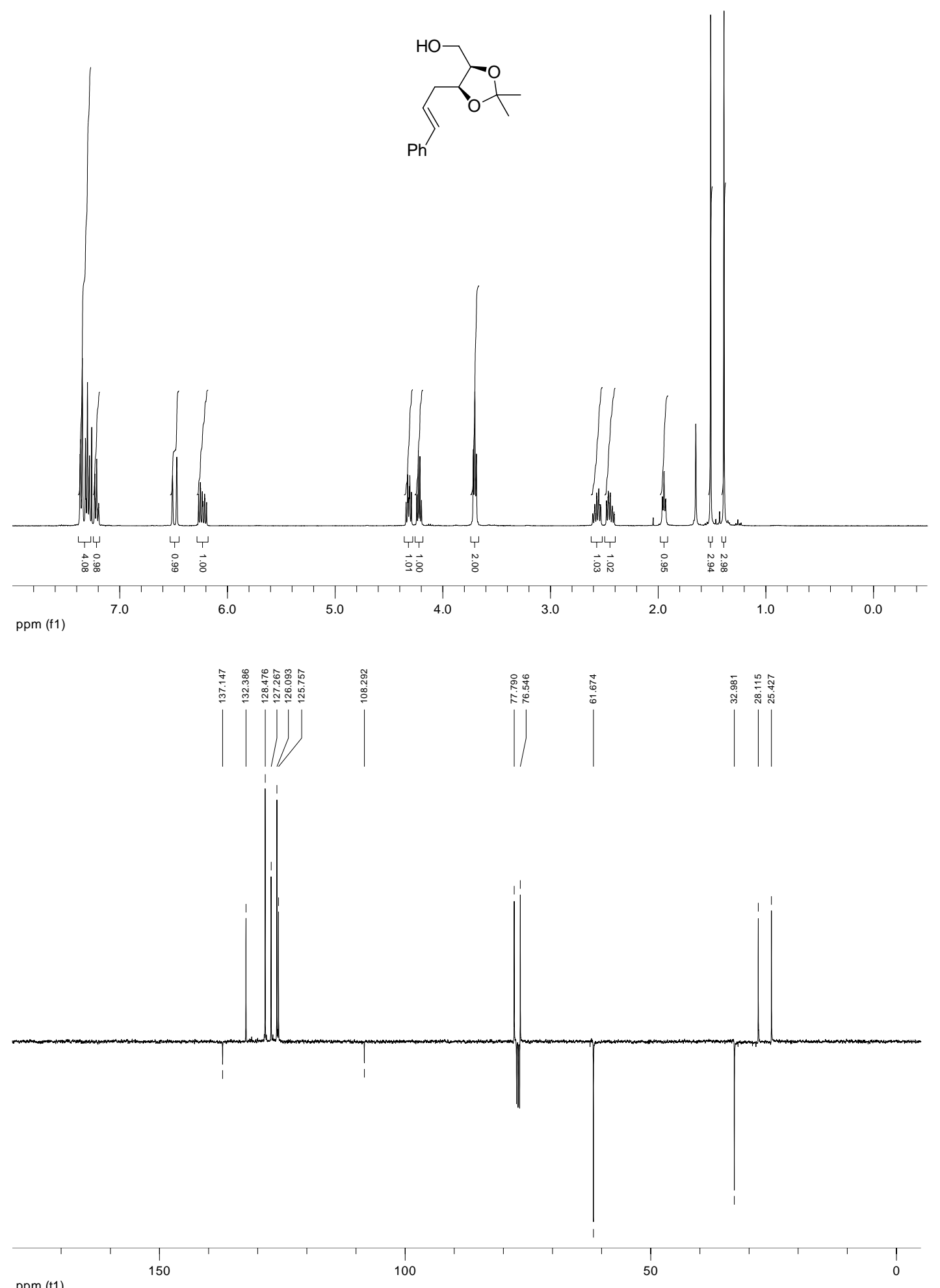

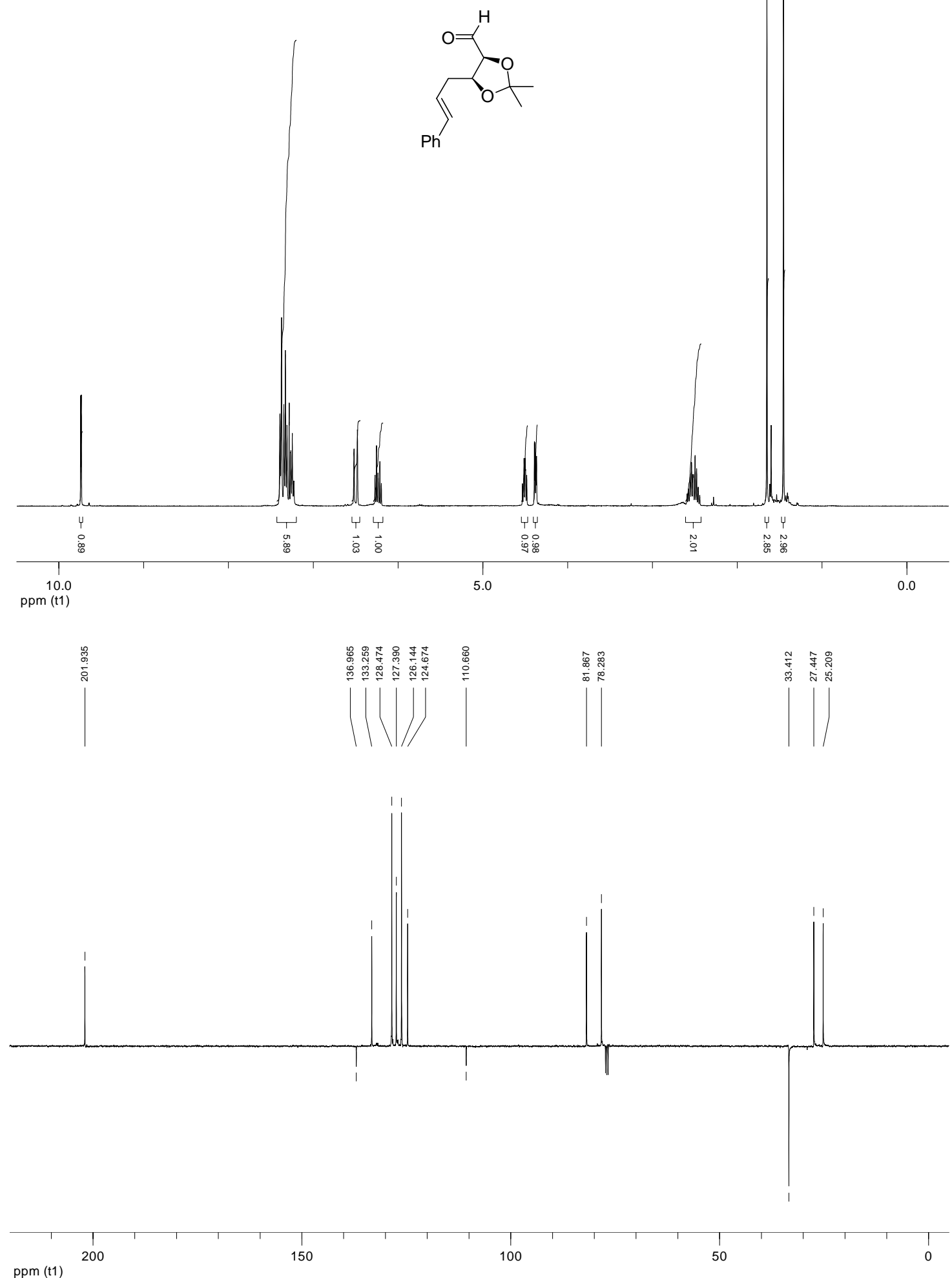

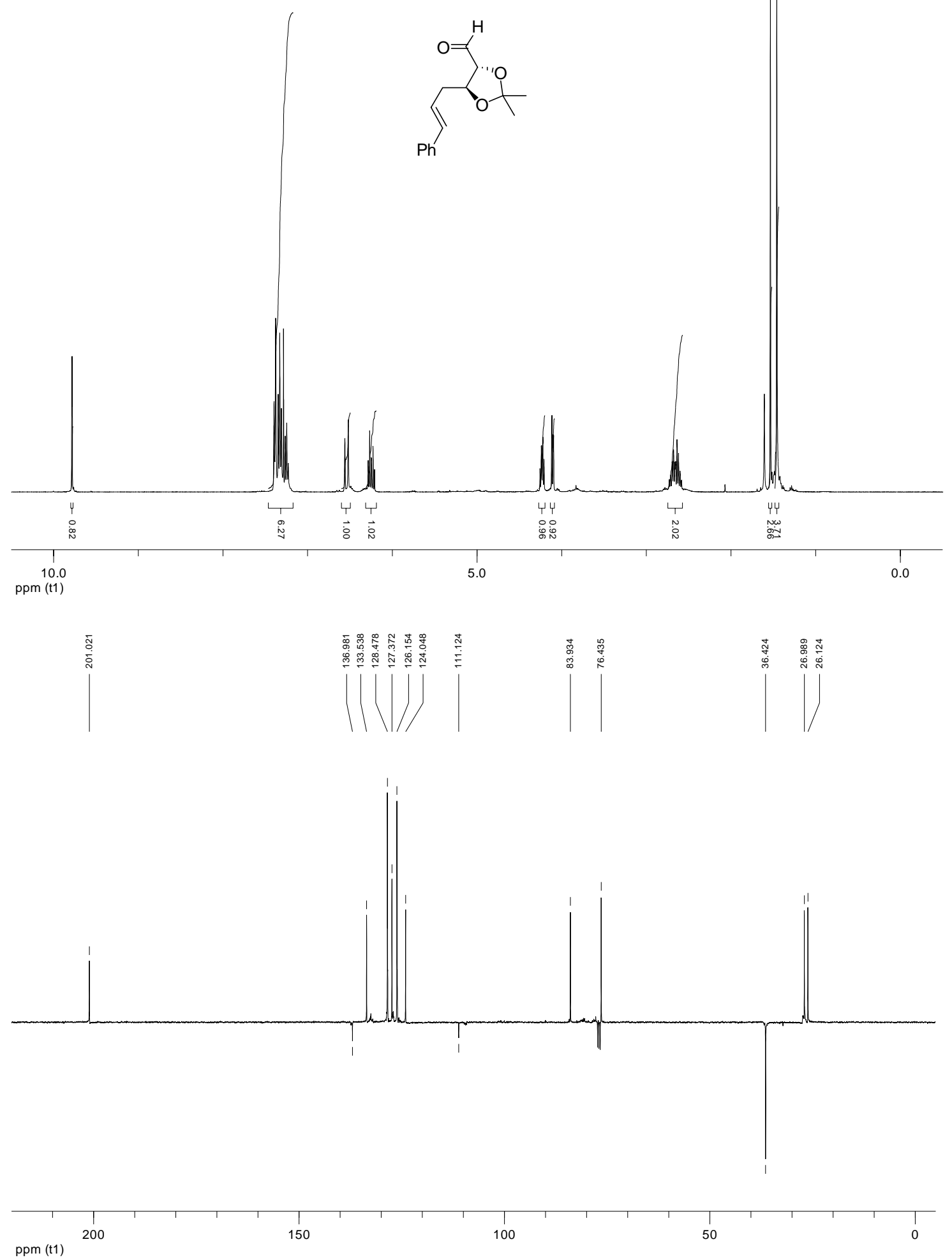

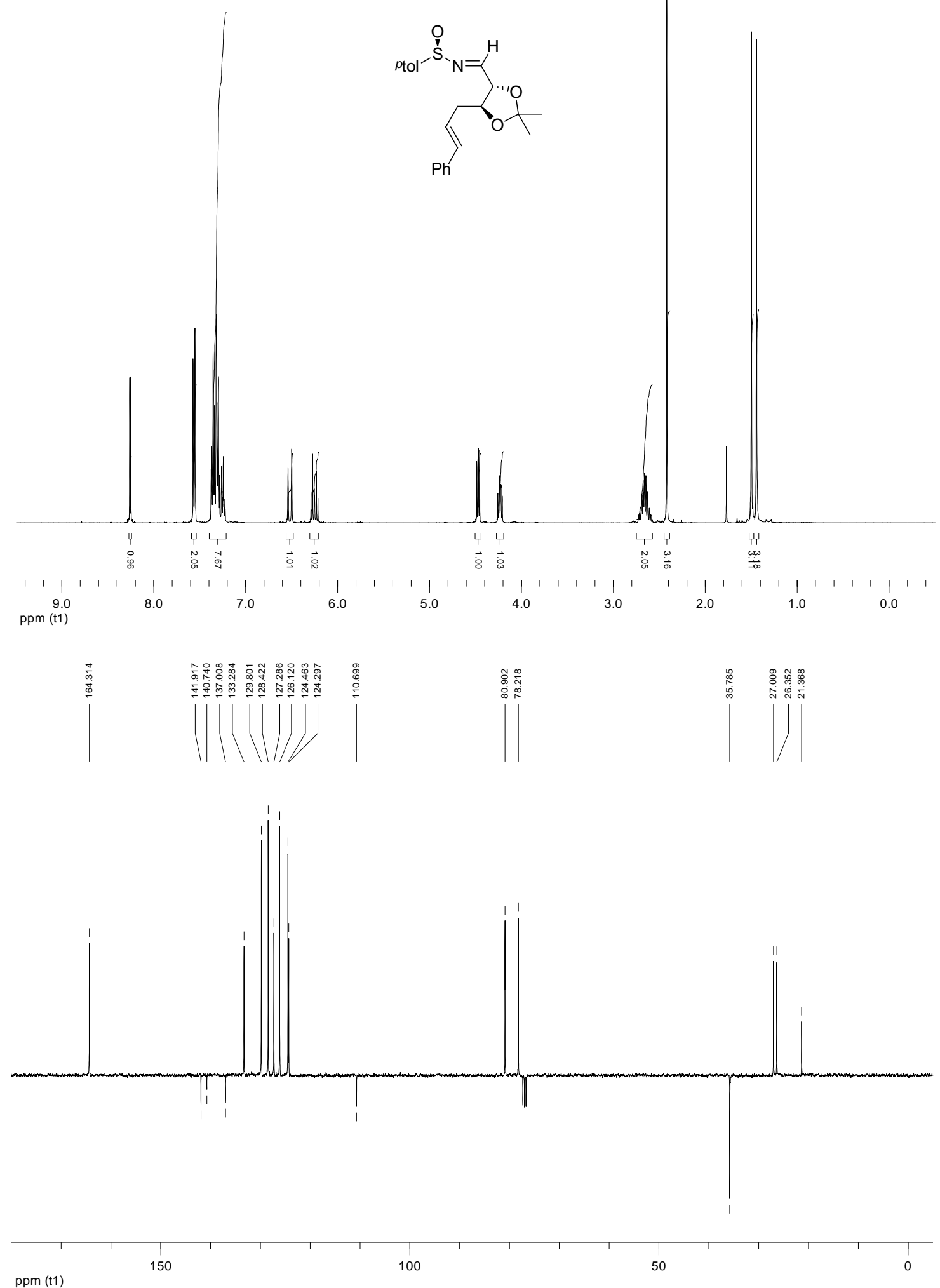


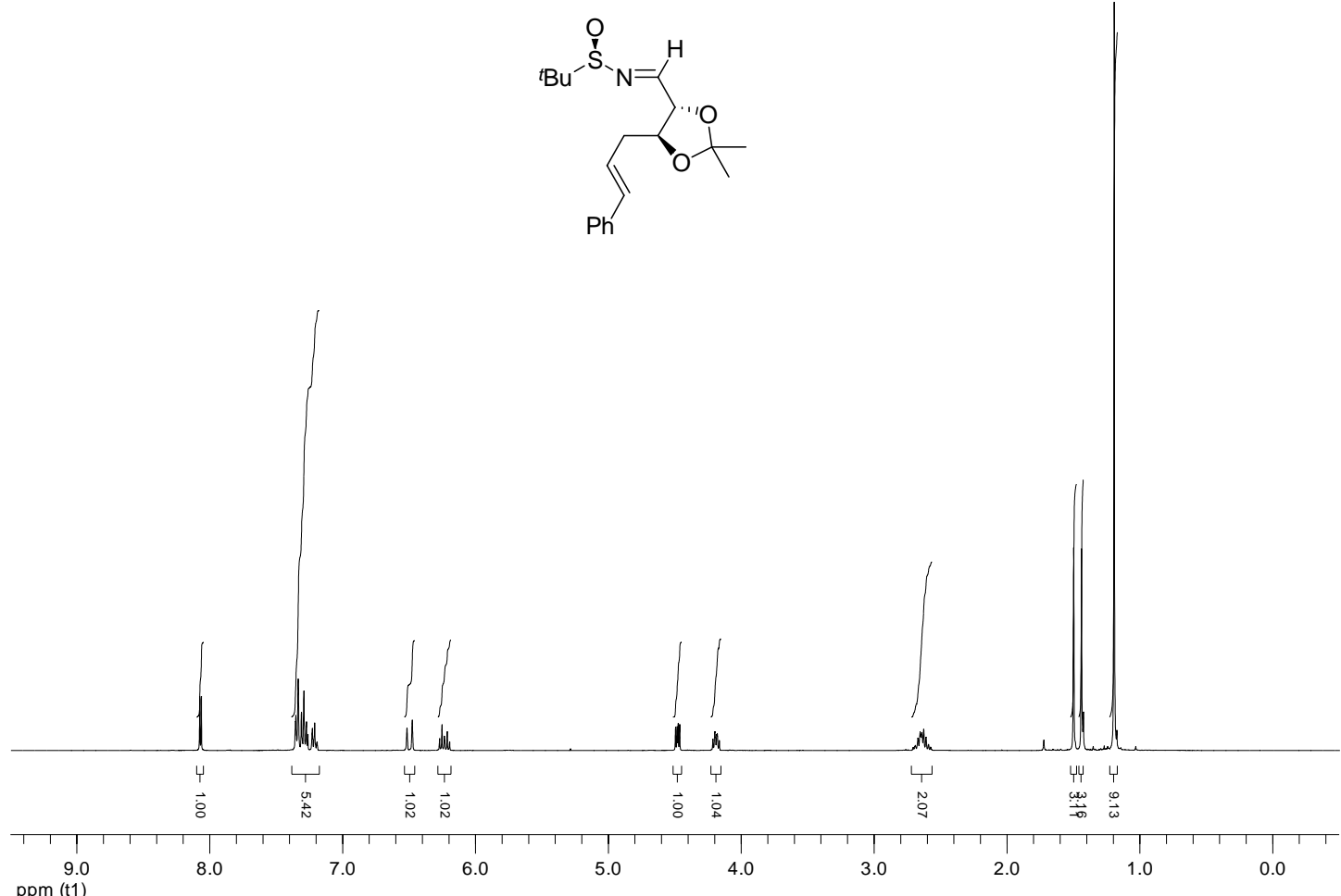
ppm (t1)
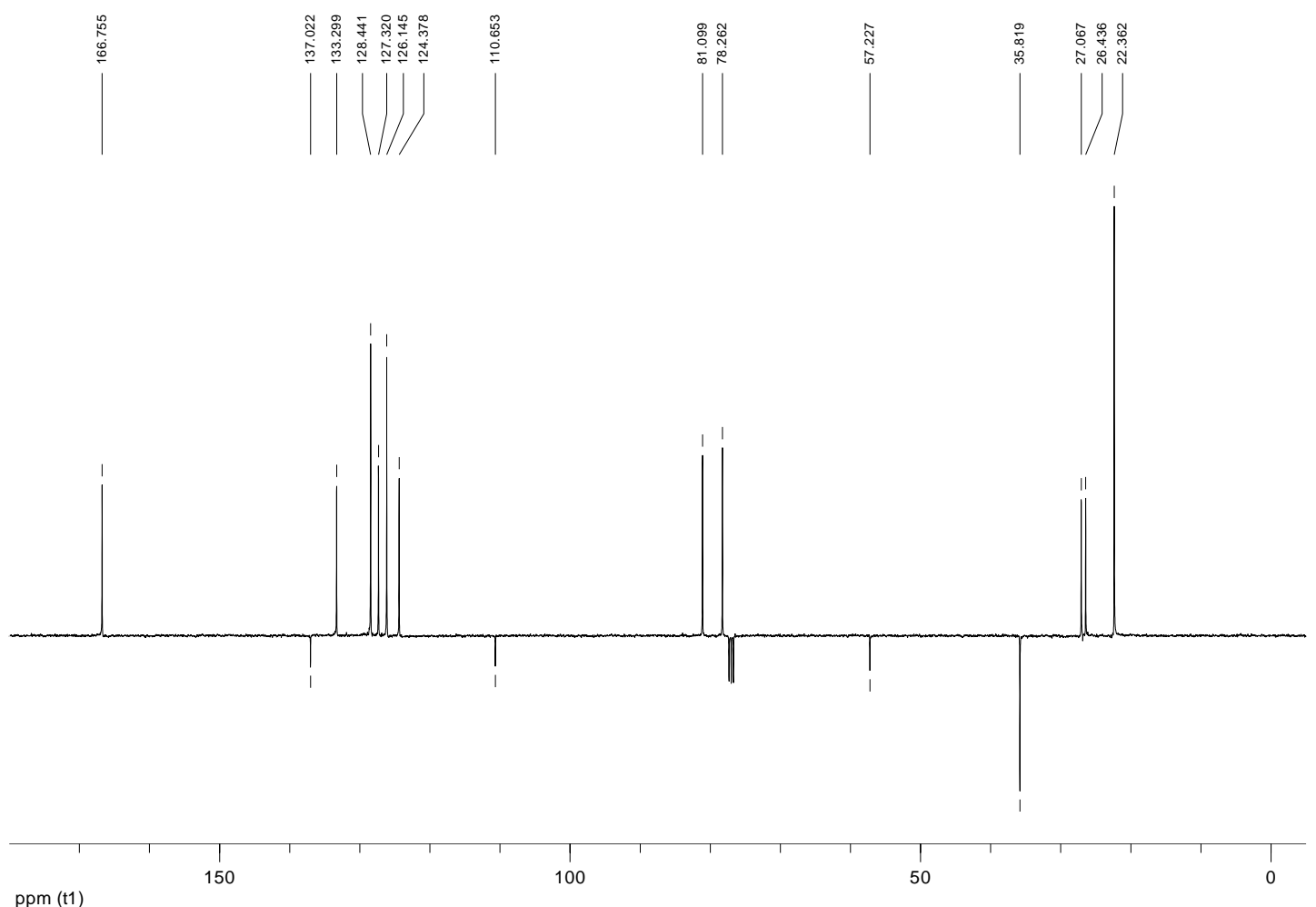

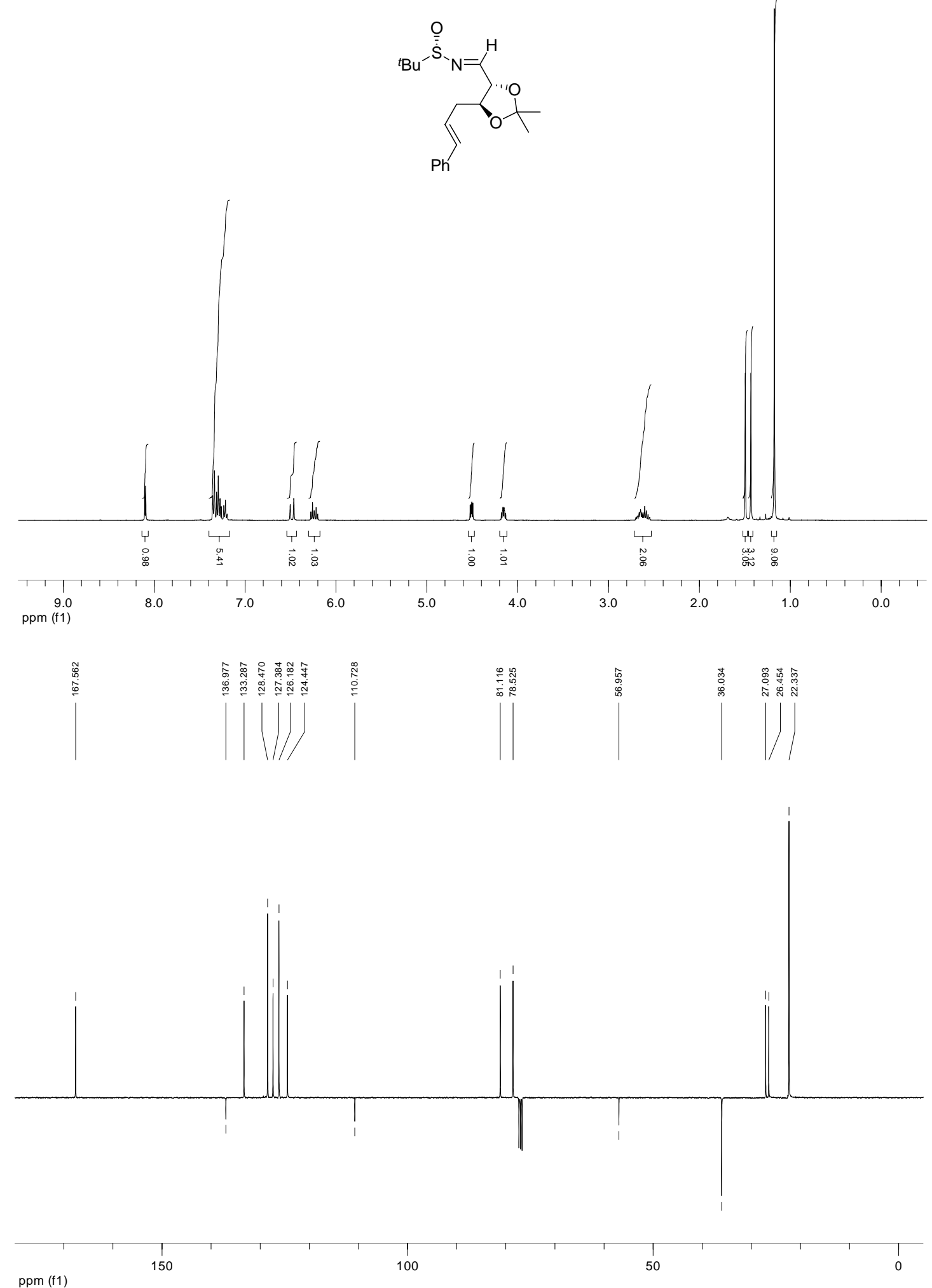


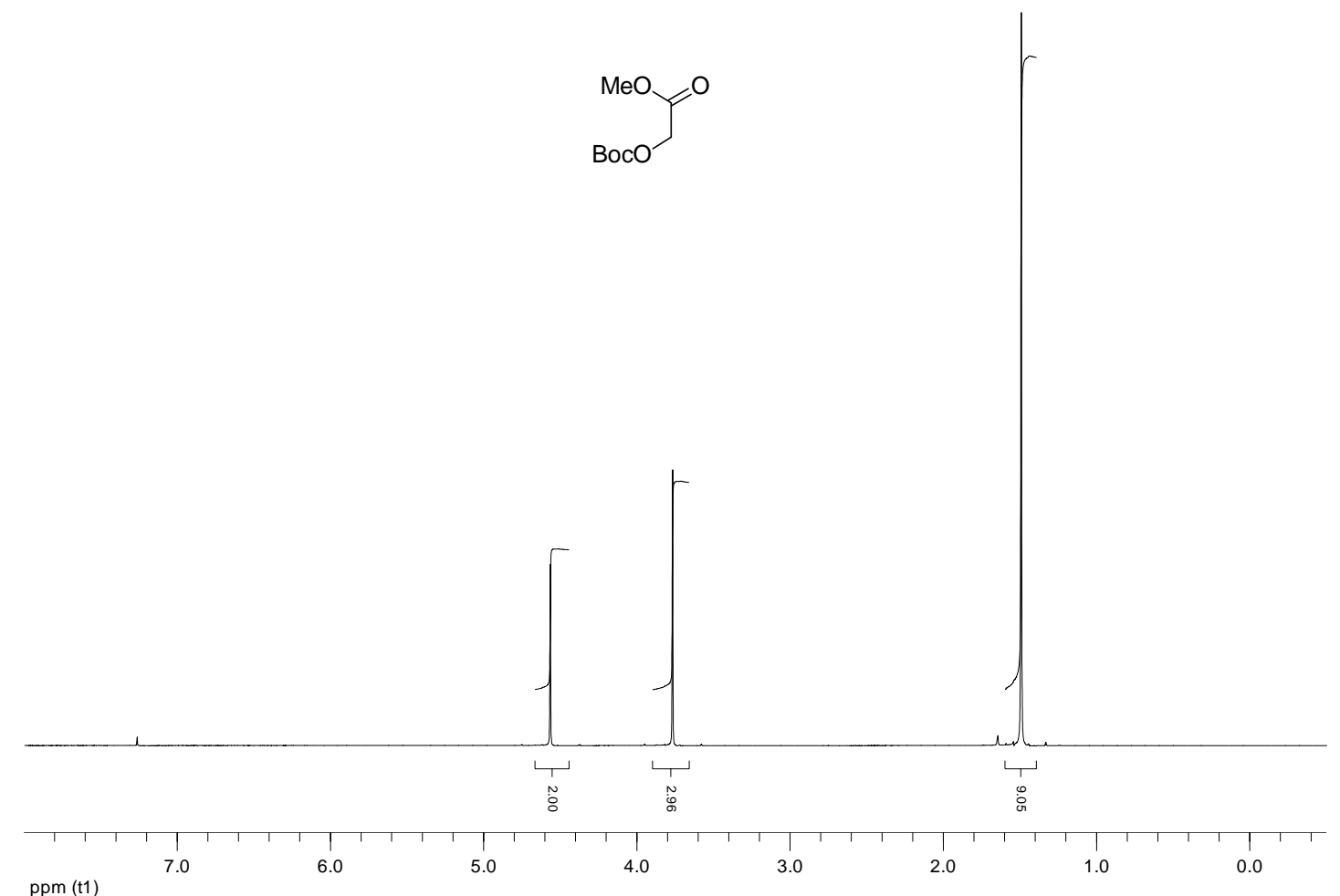

ppm (t1)

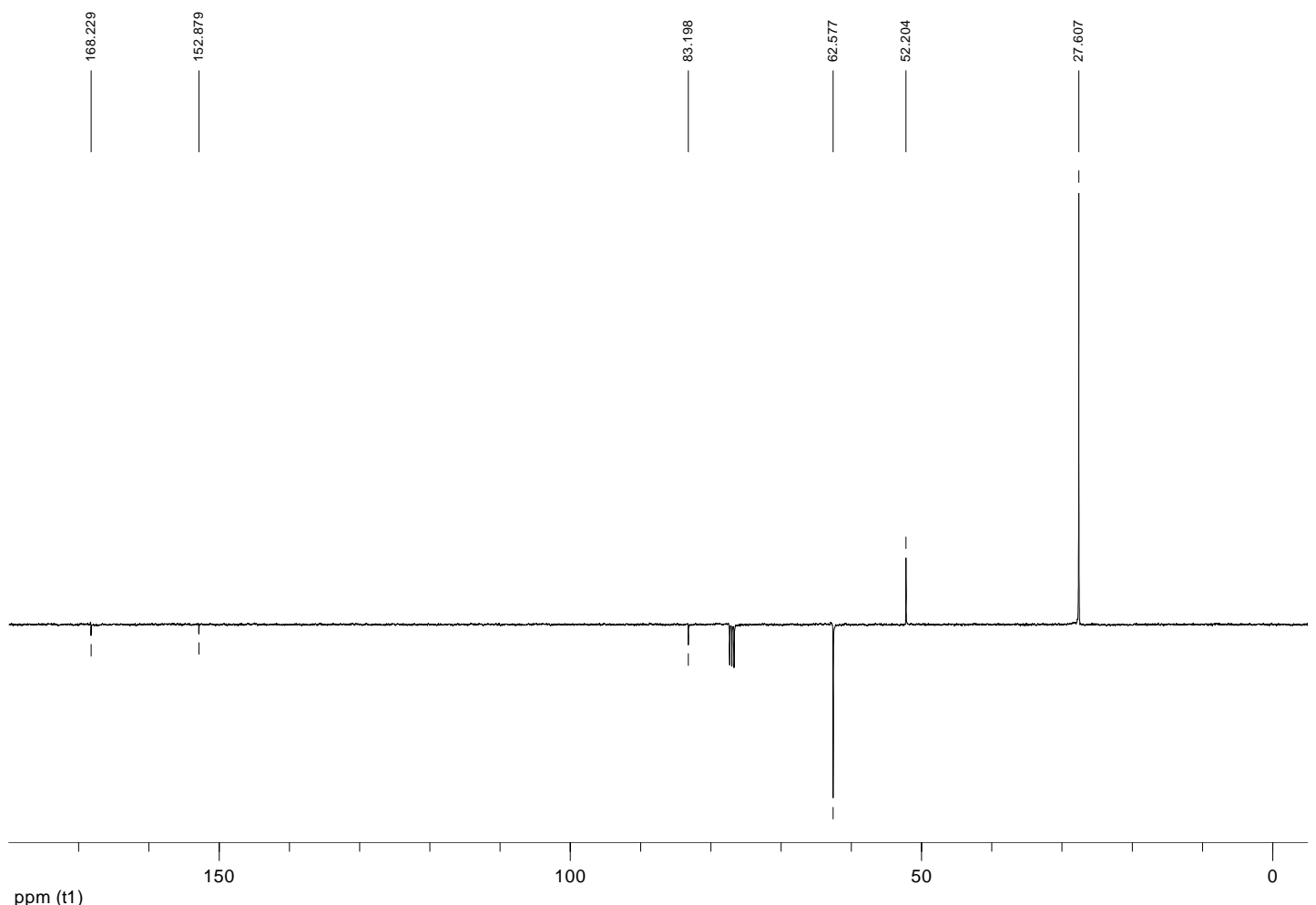




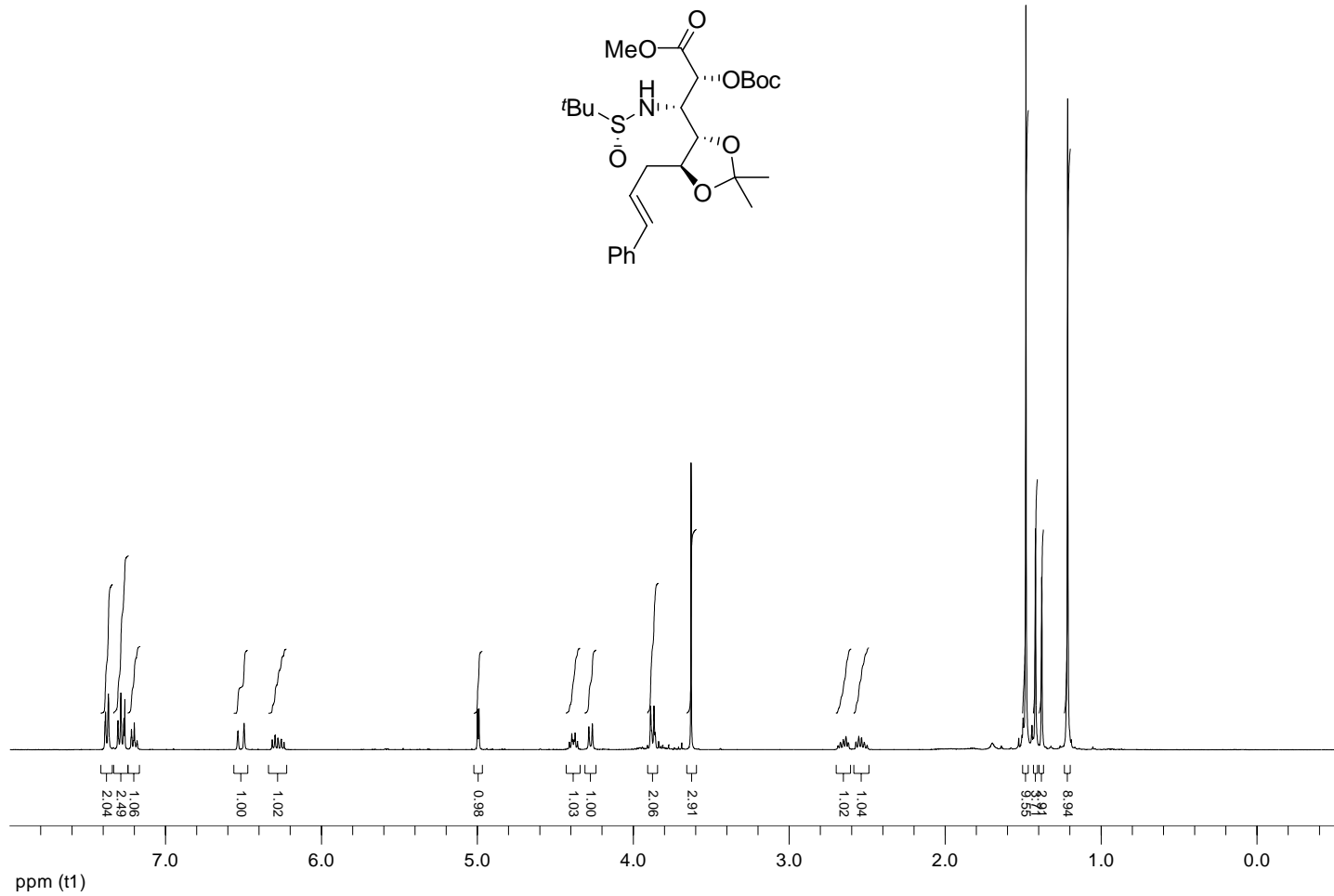

ppm (t1)
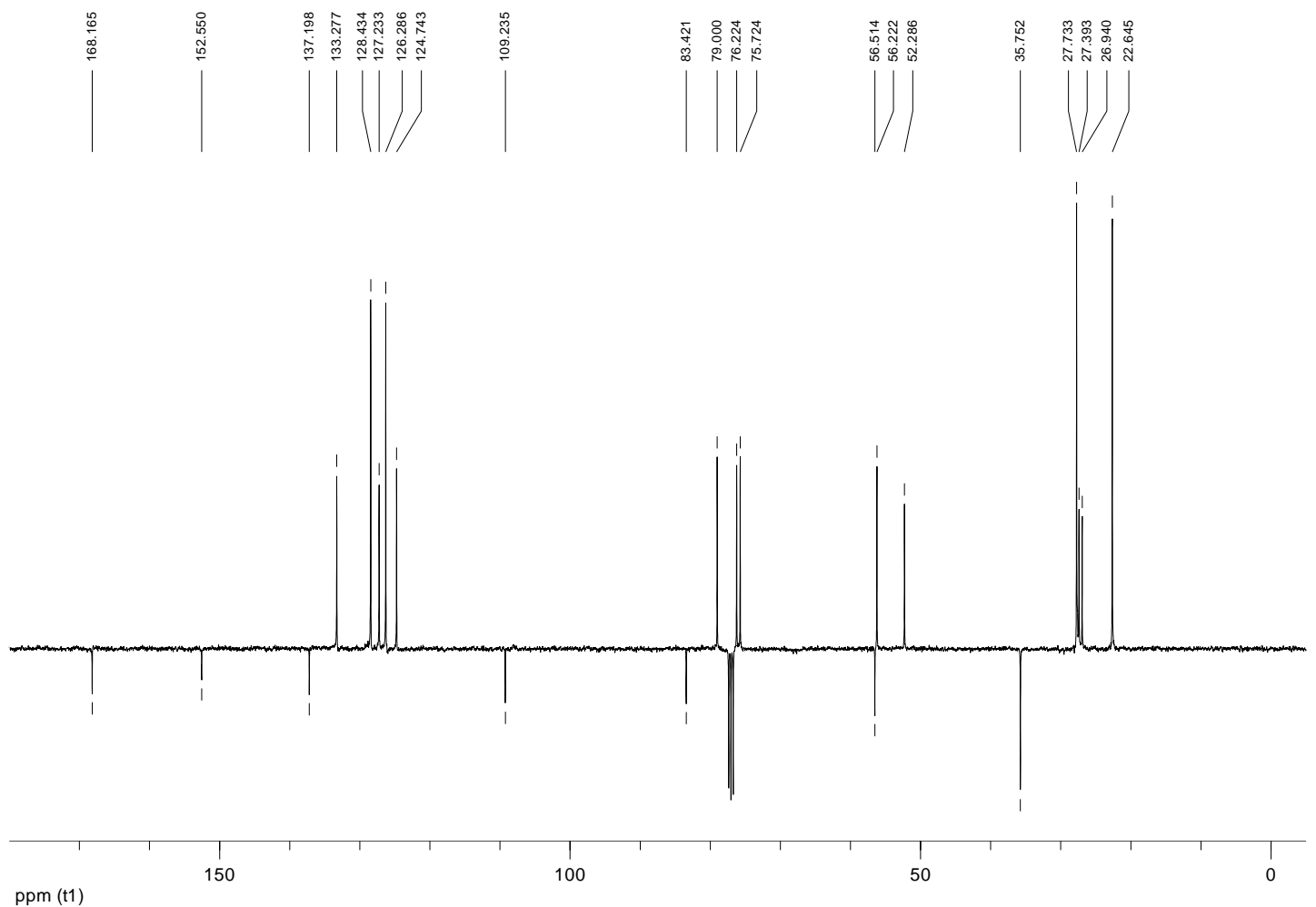


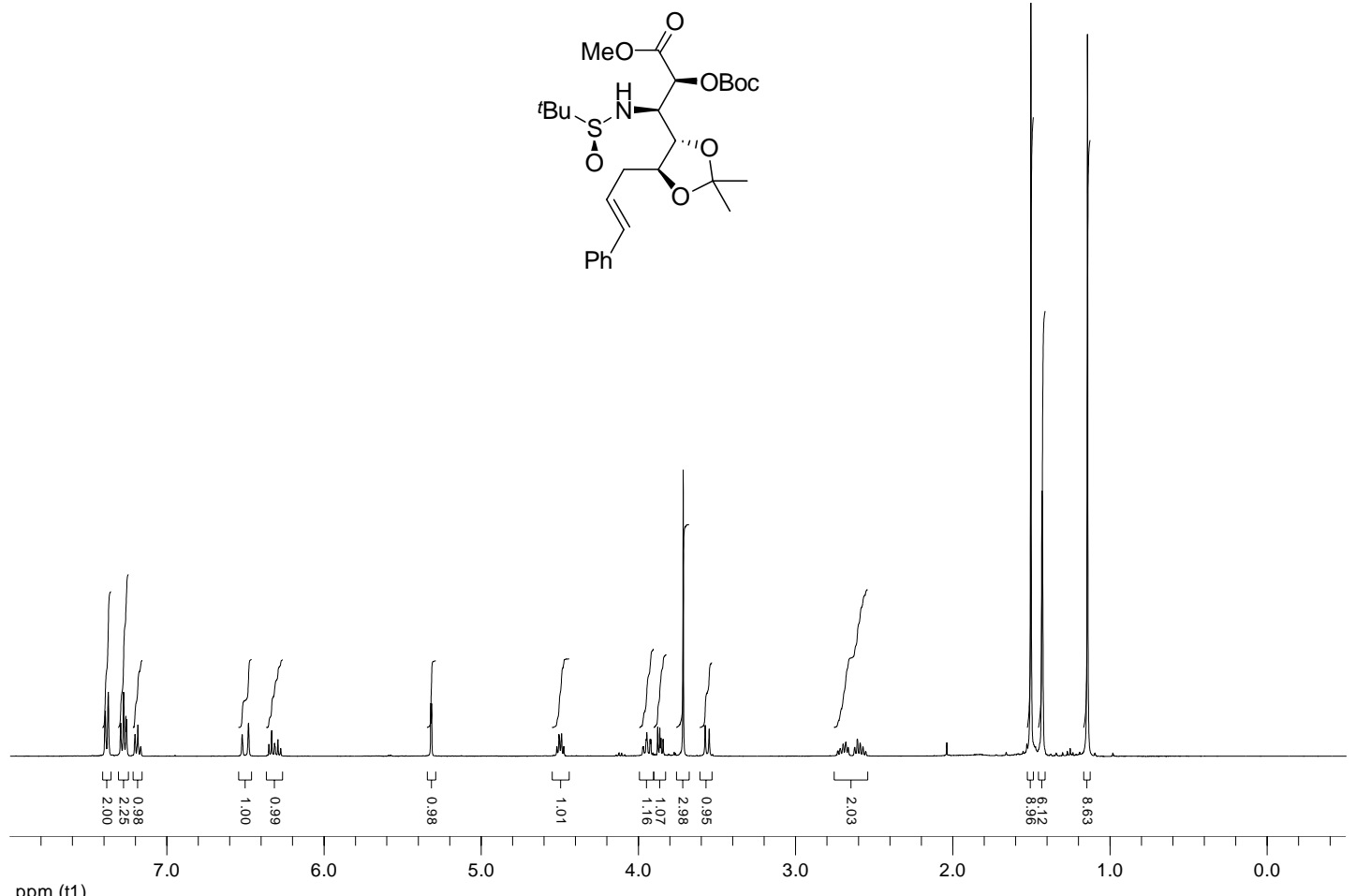

ppm (t1)
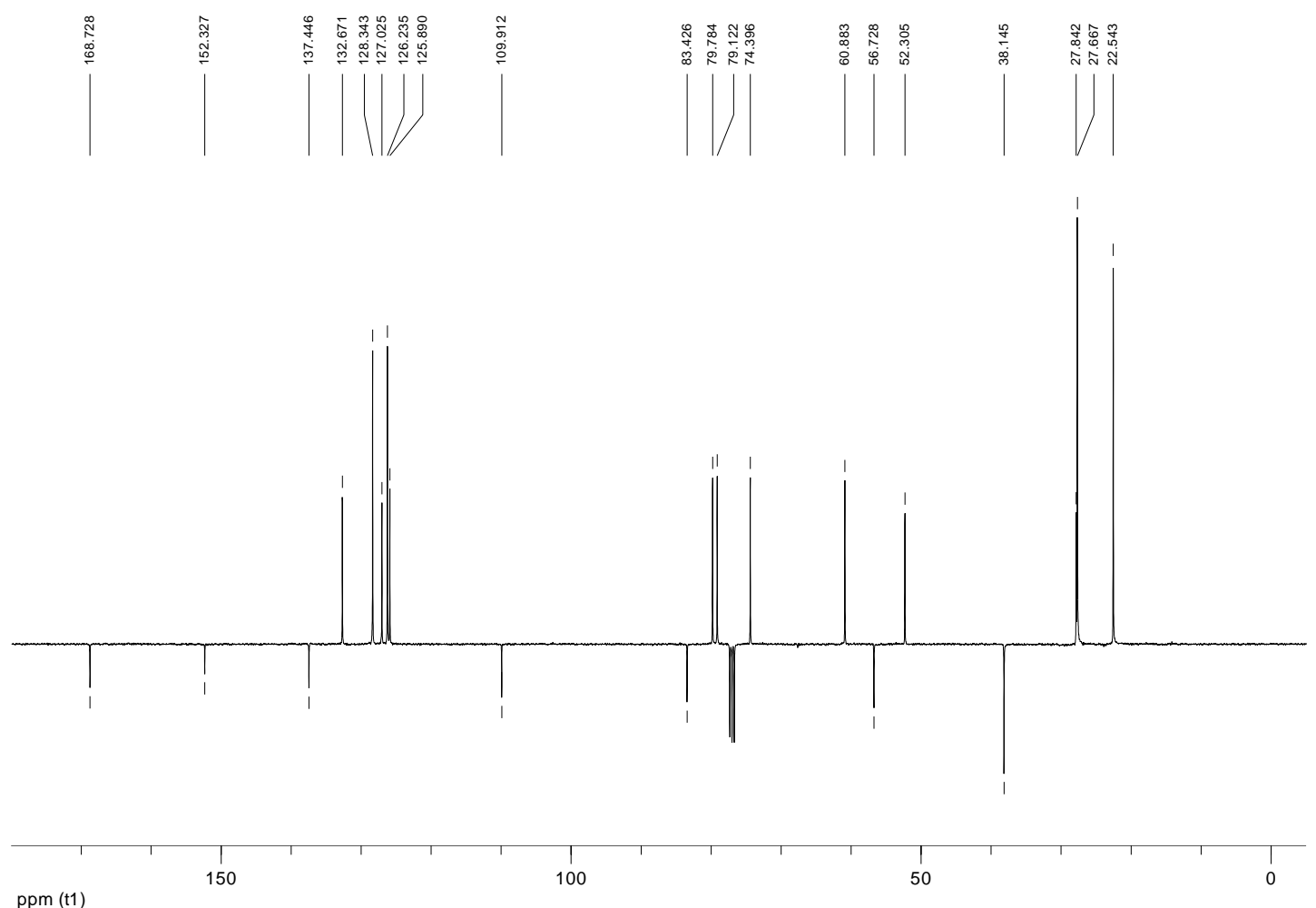

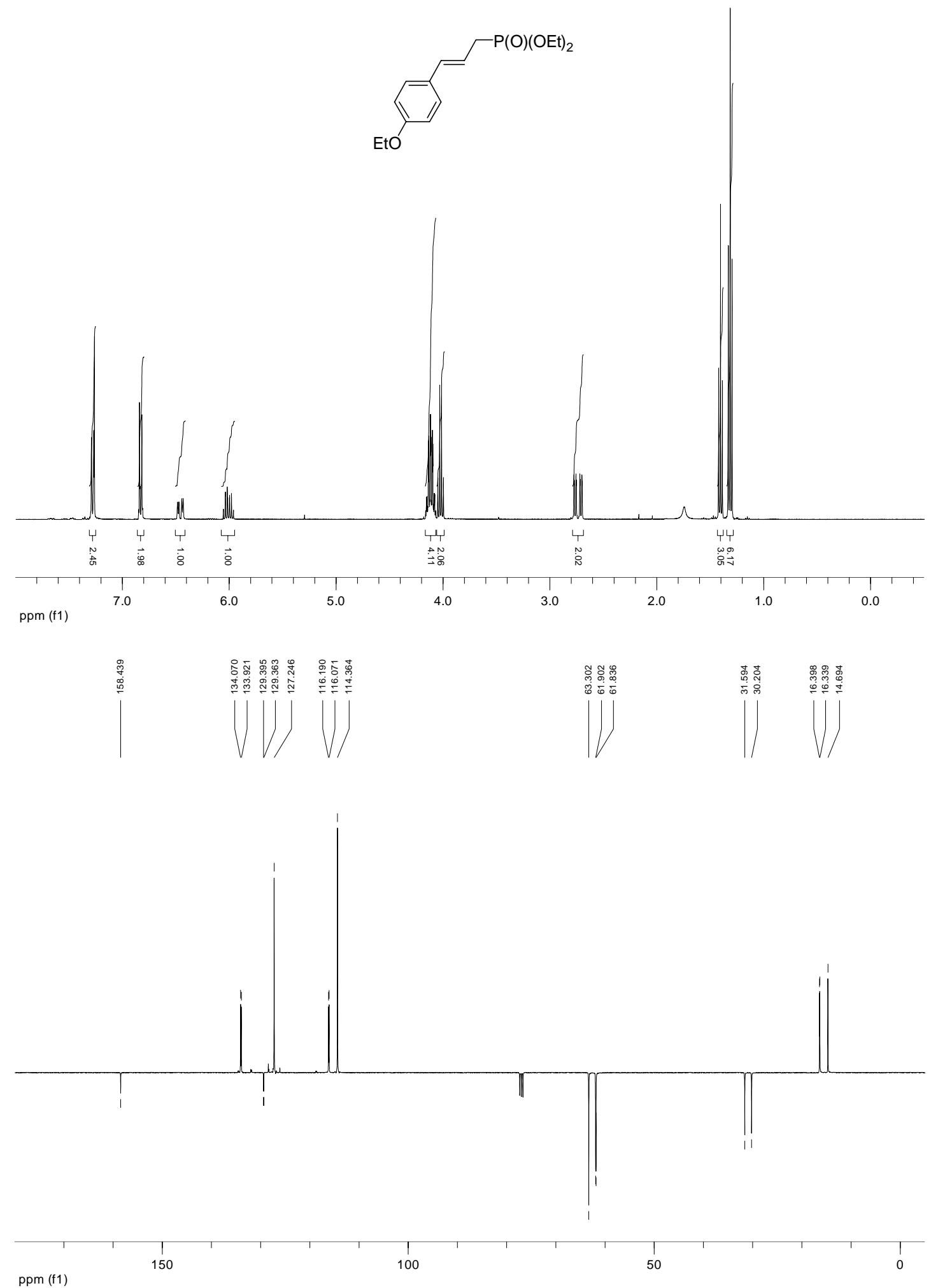


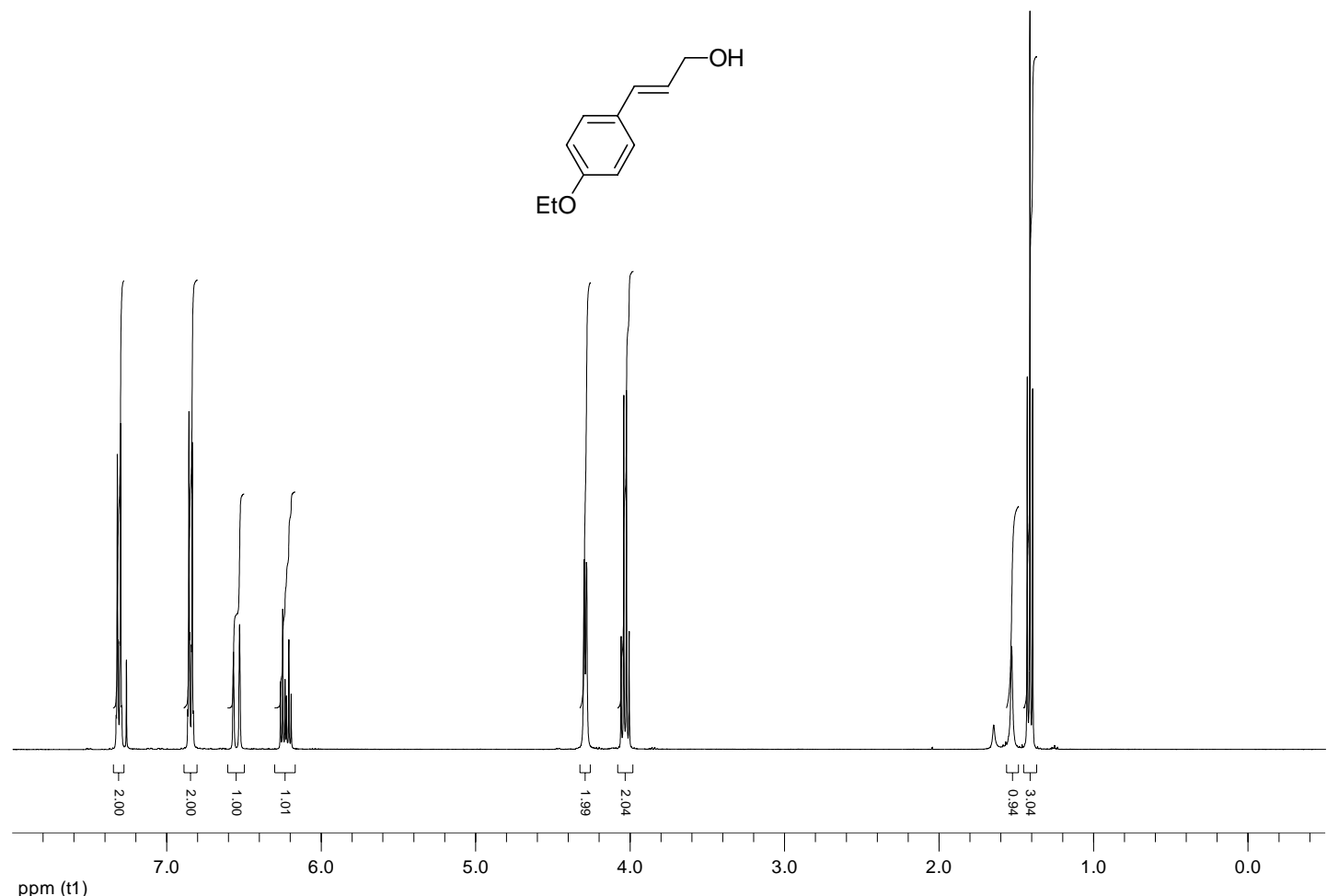

ppm (t1)
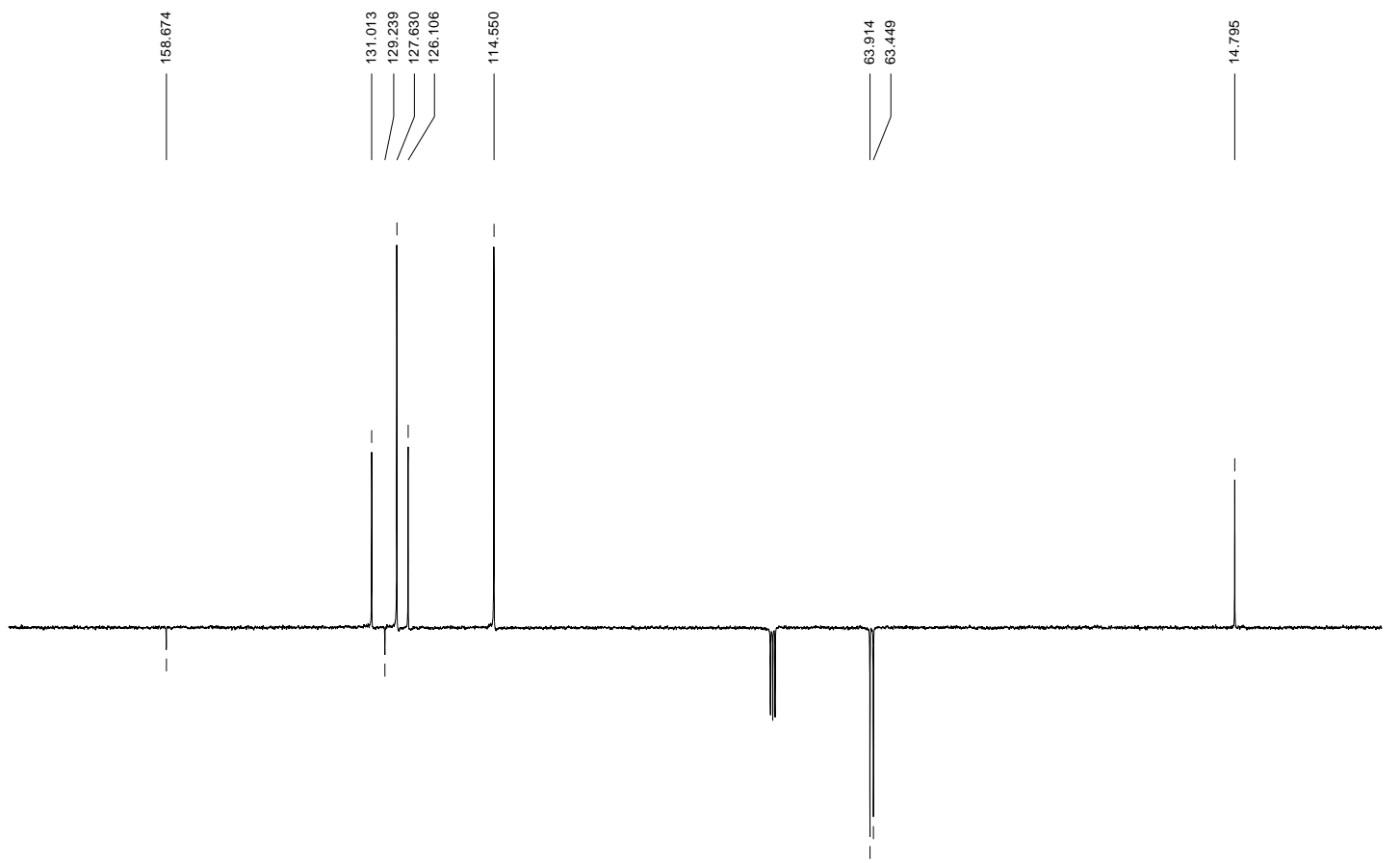


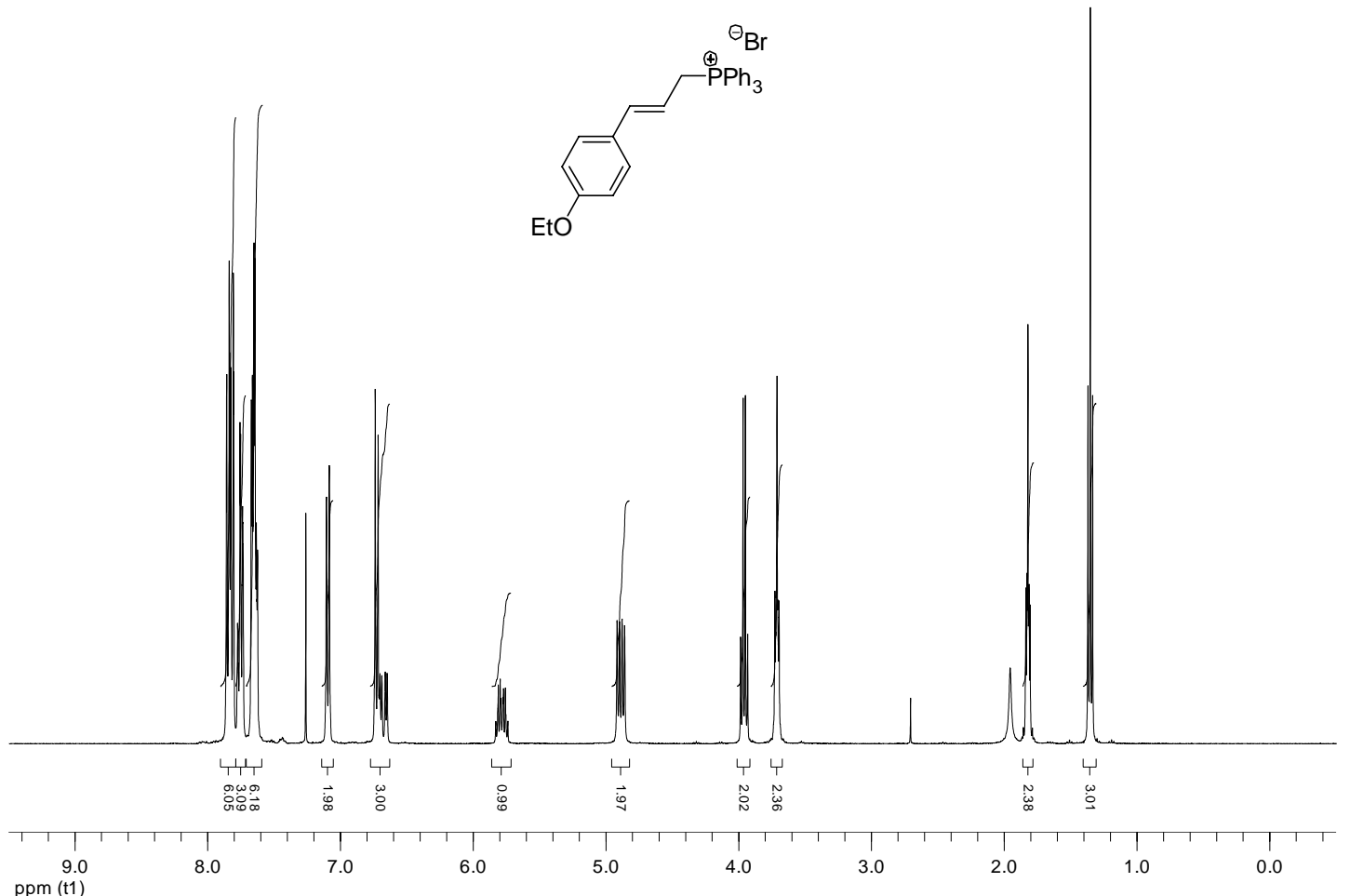

ppm (11)

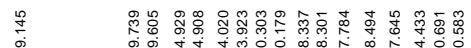

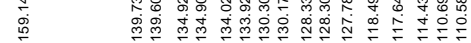

$(U$ H

离

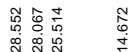
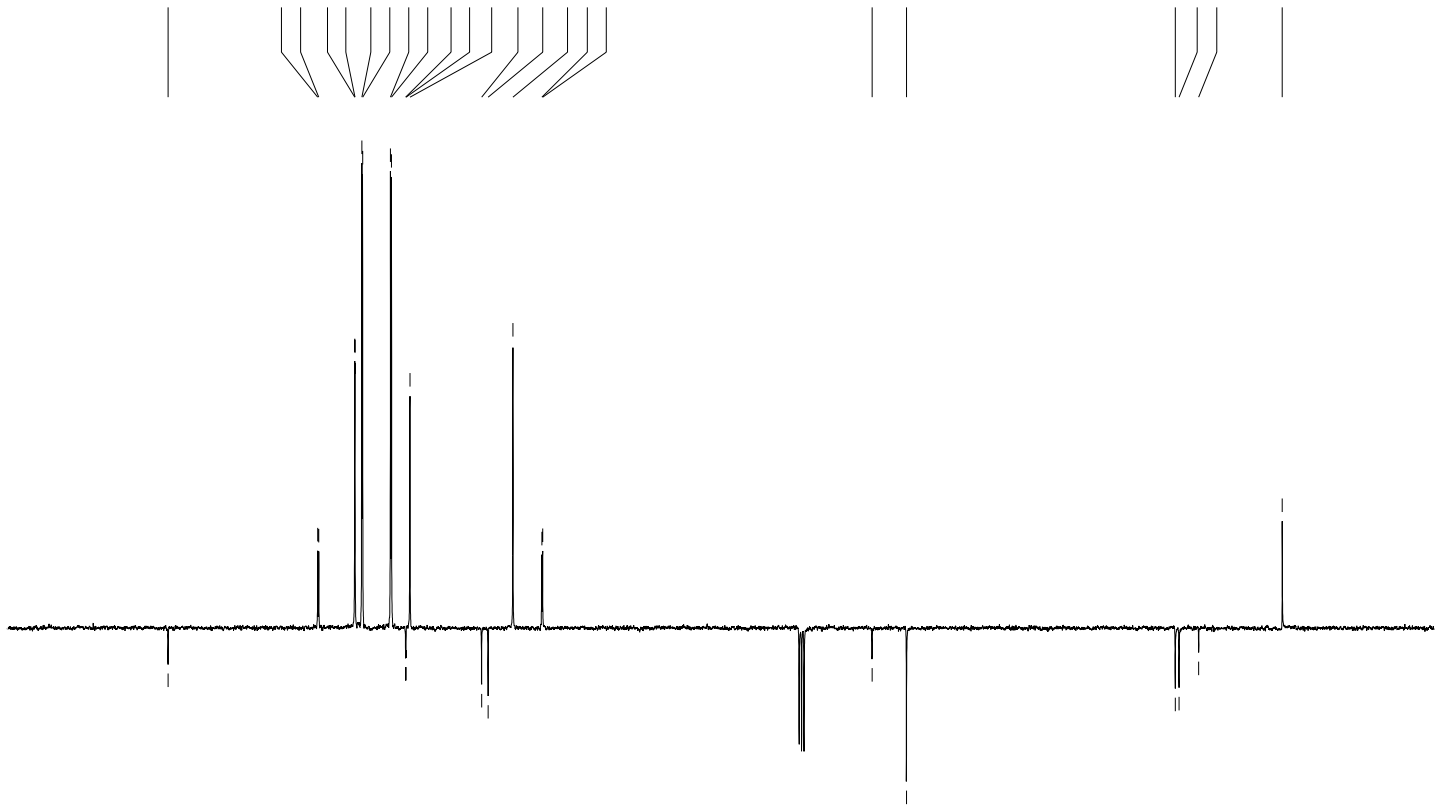


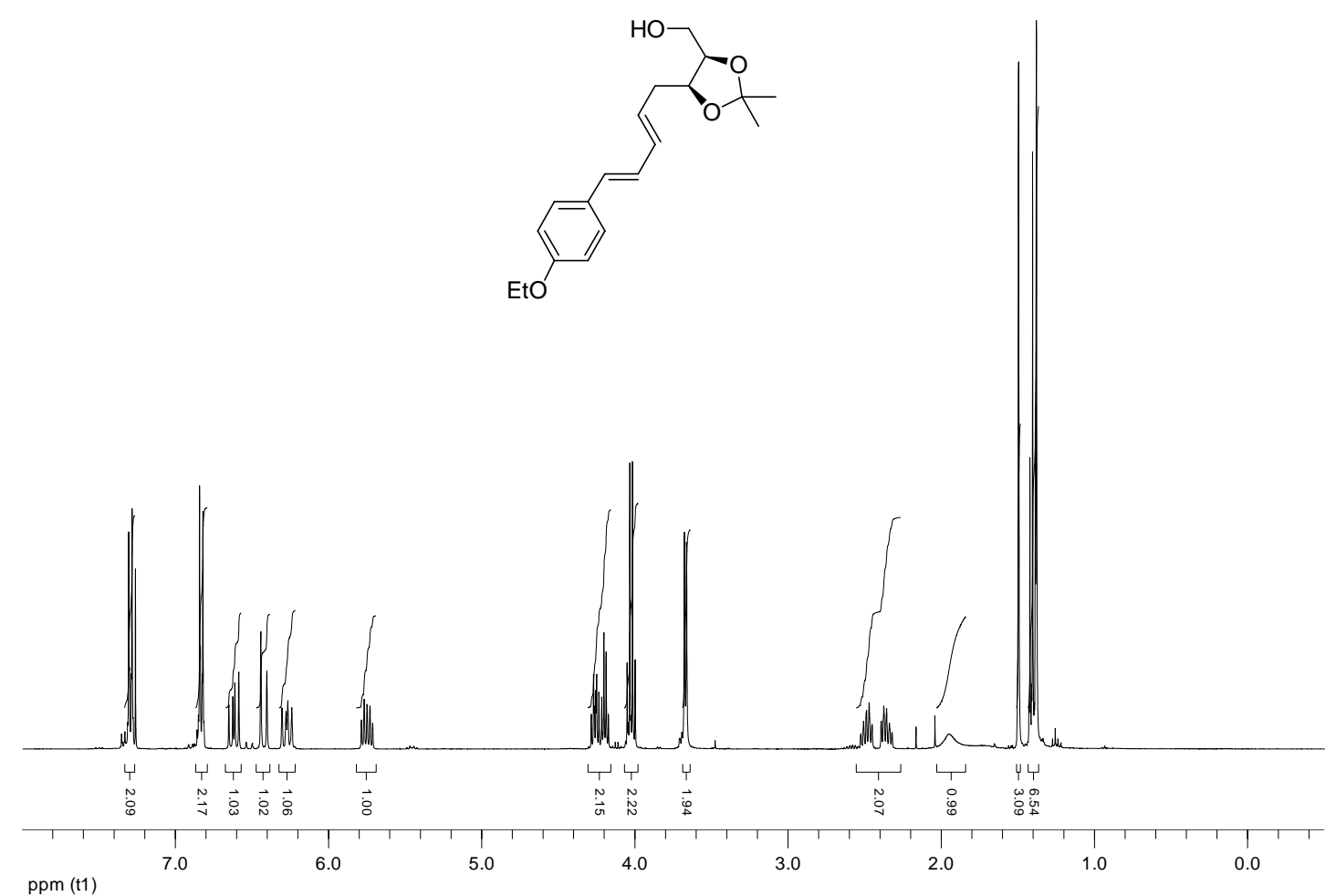

ppm (t1)
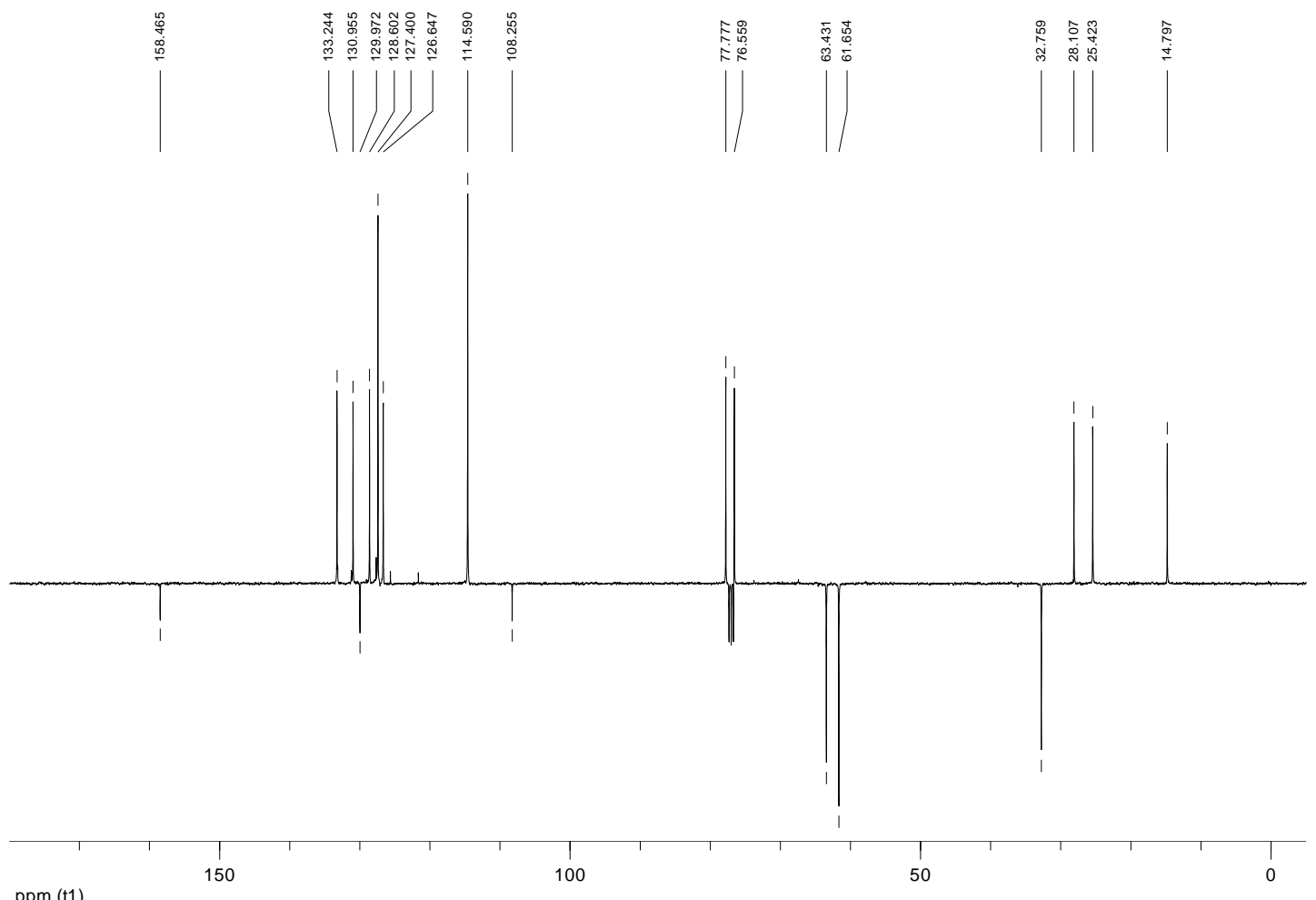


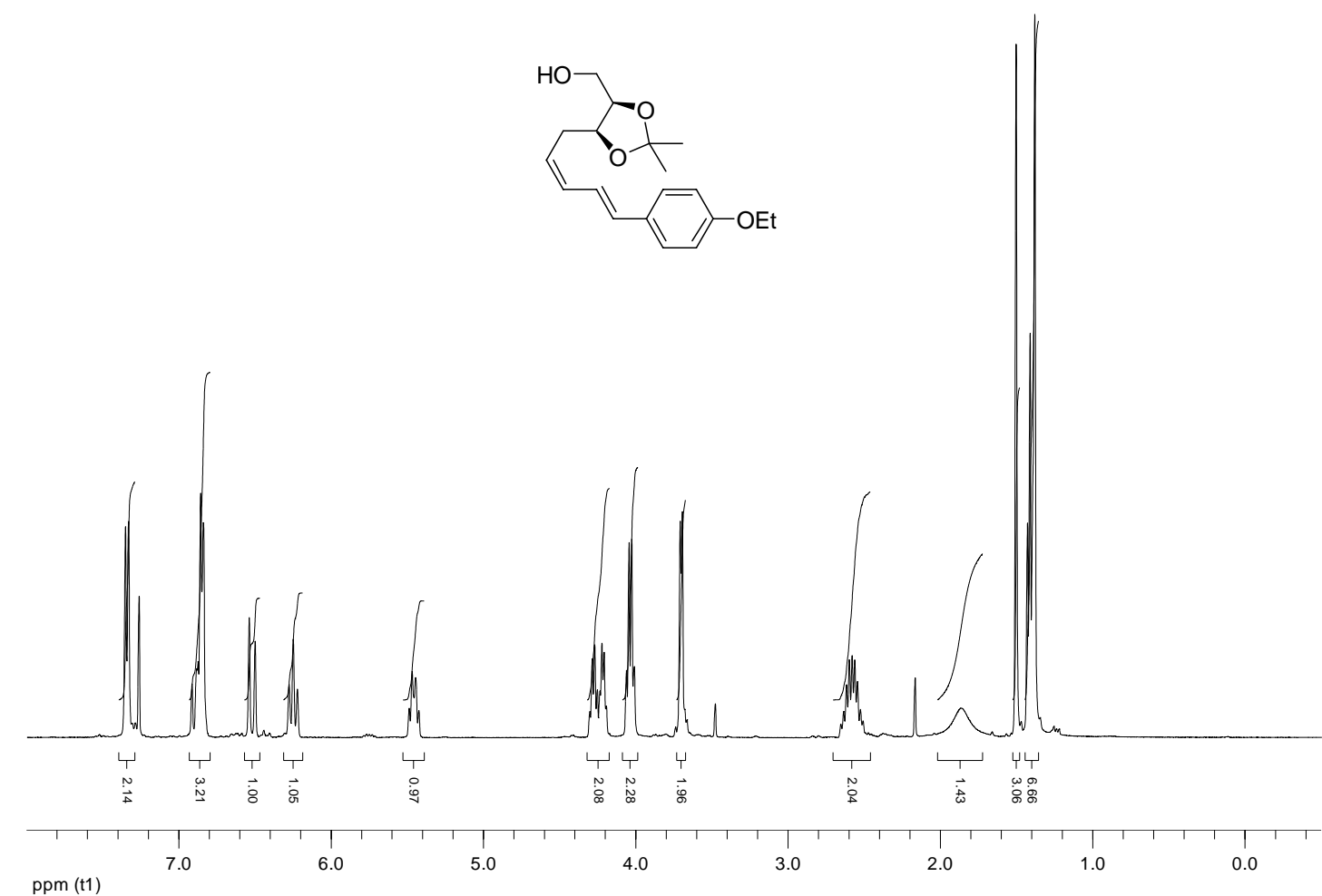

ppm (t1)
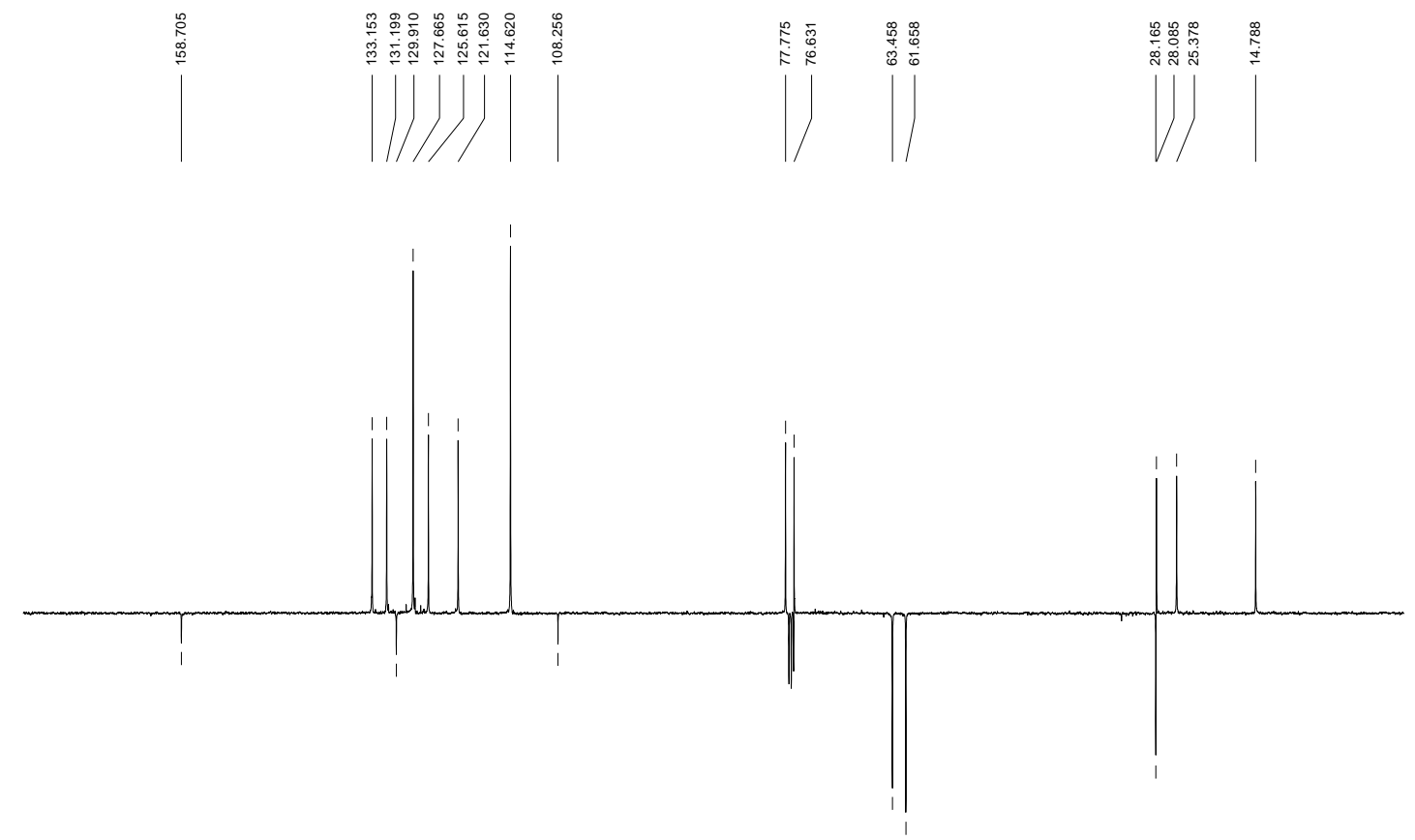

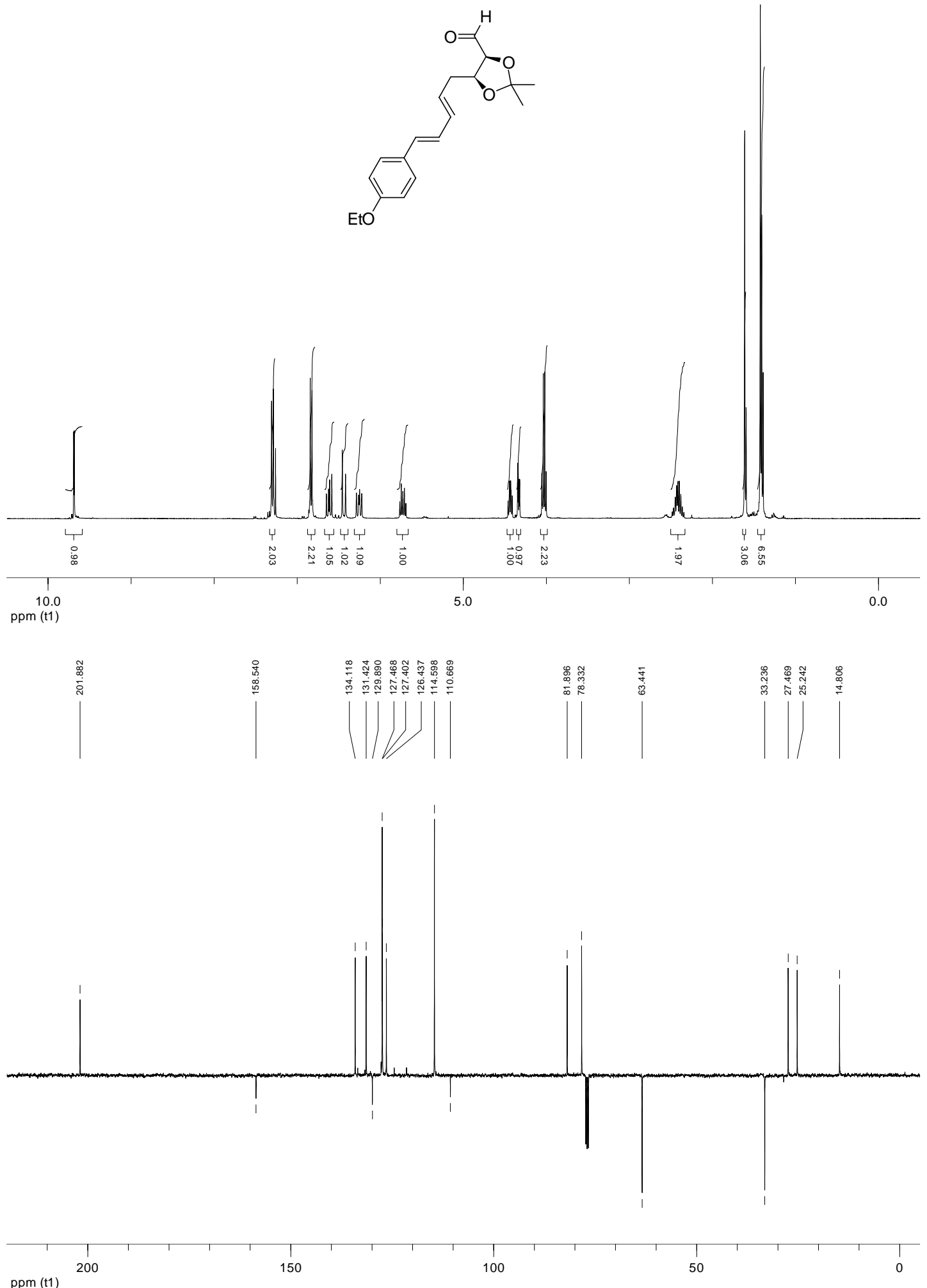


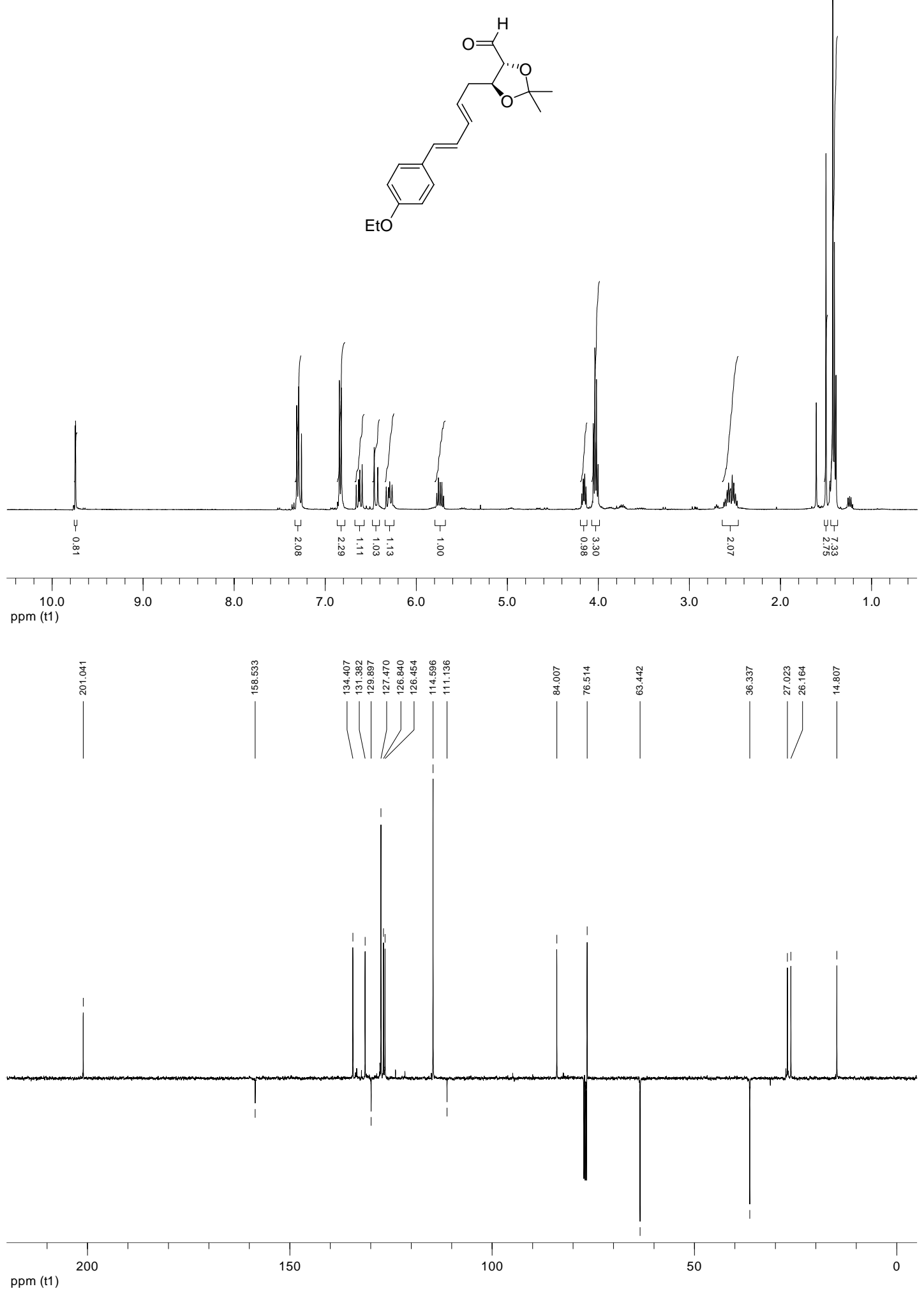



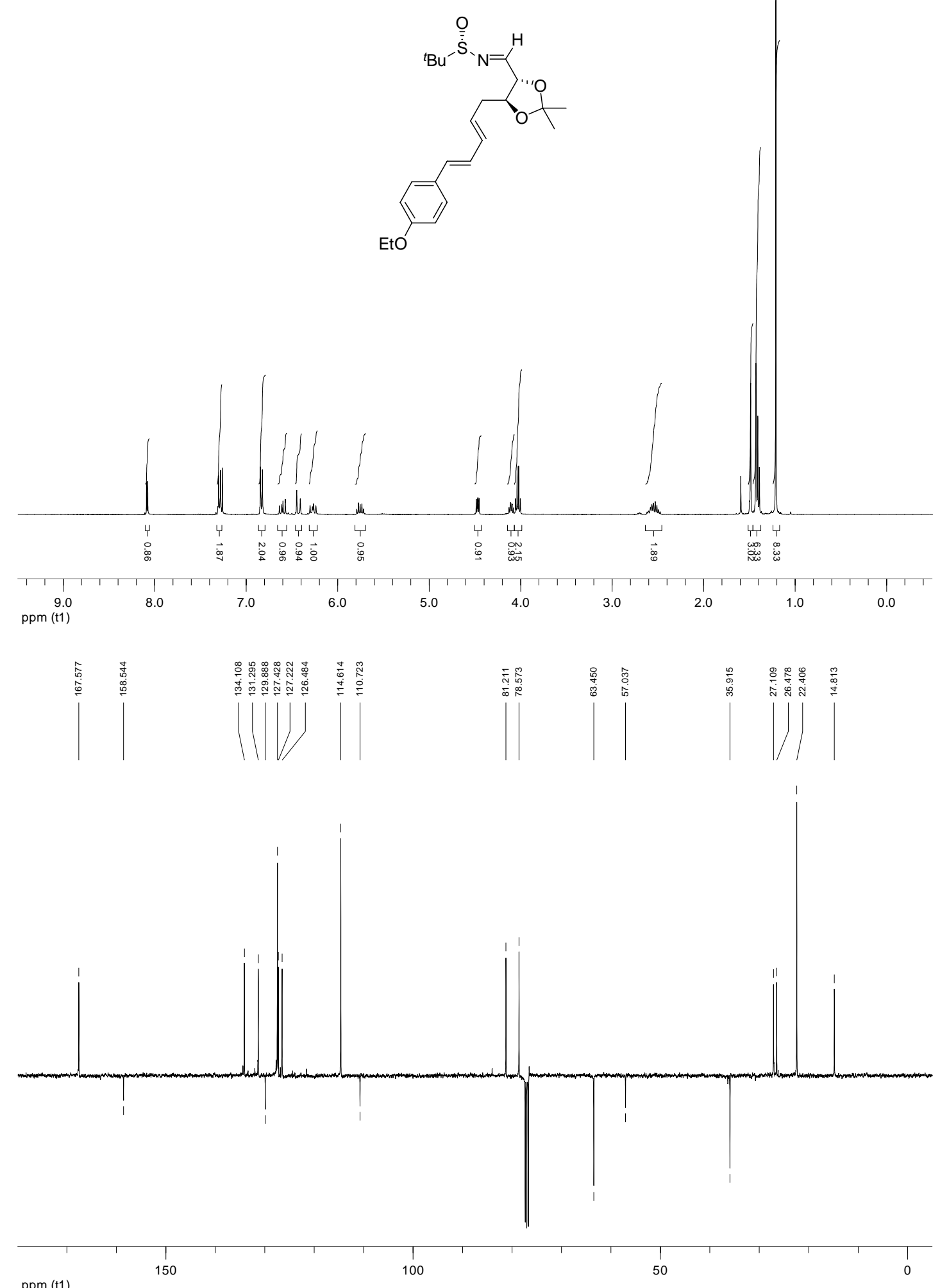


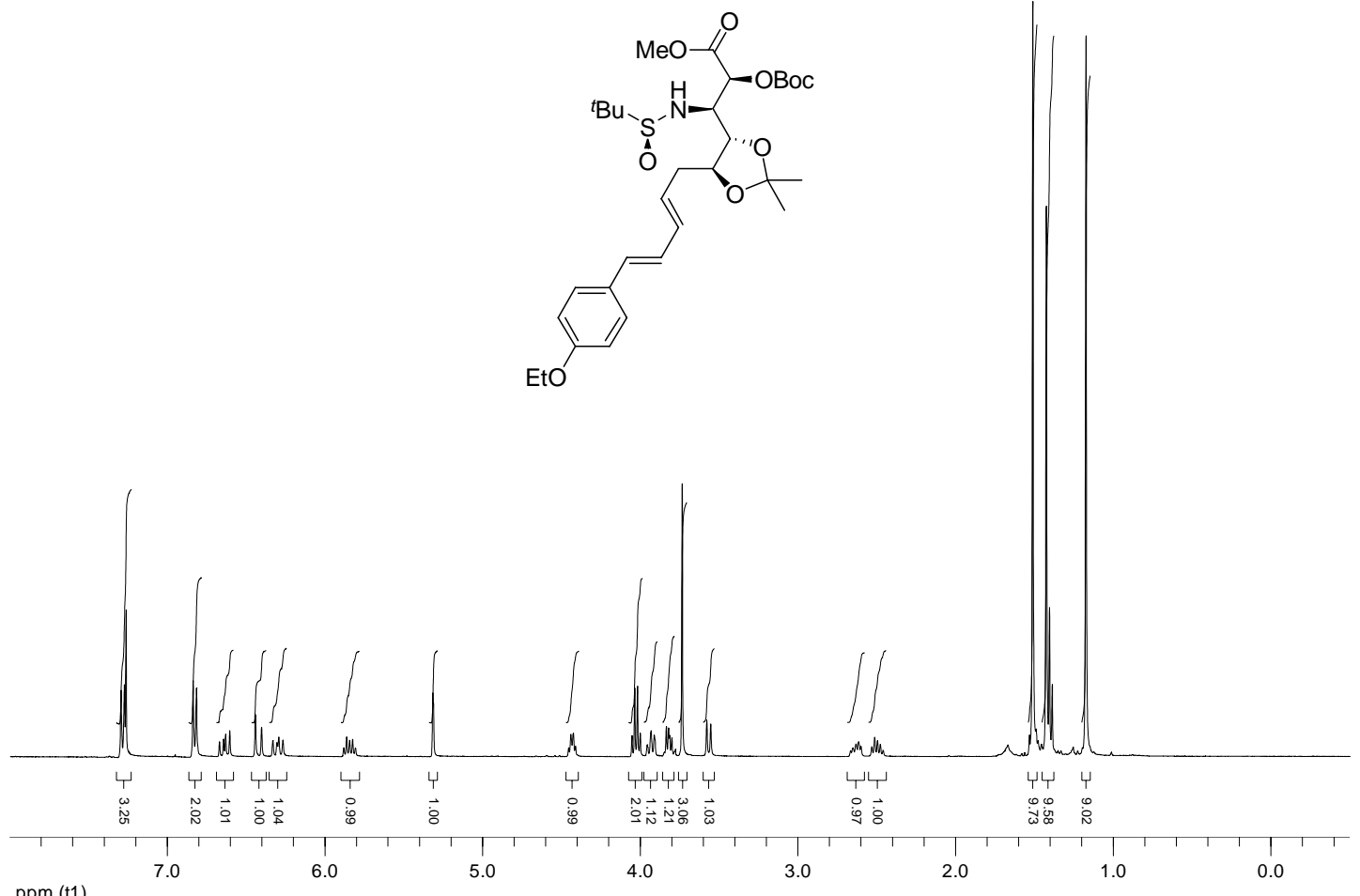

ppm (t1)
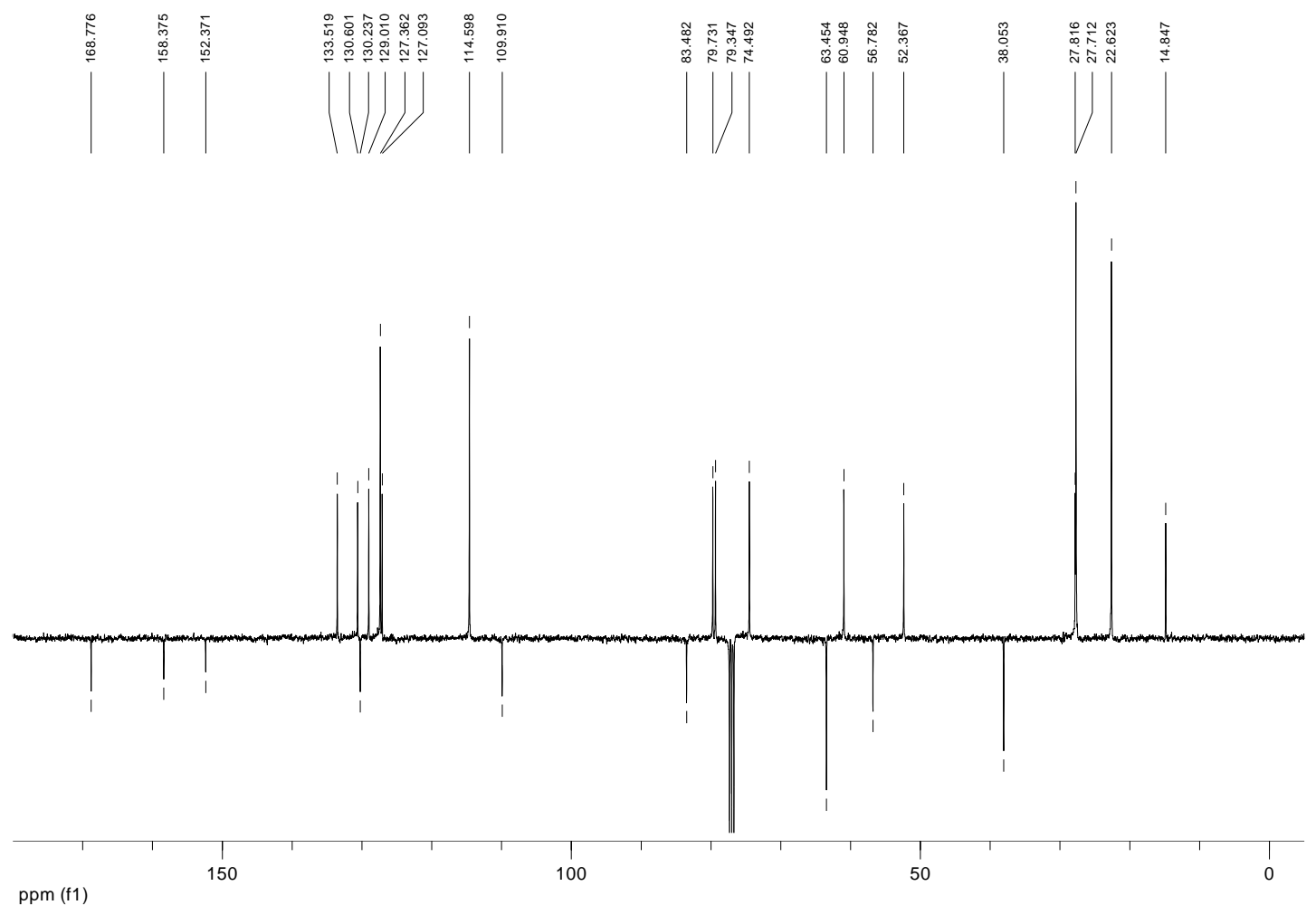


\begin{tabular}{|c|c|c|}
\hline compound & $7 \mathbf{b}$ & 7c \\
\hline formula & $\mathrm{C}_{27} \mathrm{H}_{41} \mathrm{NO}_{8} \mathrm{~S}$ & $\mathrm{C}_{27} \mathrm{H}_{41} \mathrm{NO}_{8} \mathrm{~S}$ \\
\hline Fw & 539.67 & 539.67 \\
\hline crystal system & orthorhombic & triclinic \\
\hline space group & P 212121 & $\mathrm{P} 1$ \\
\hline $\mathrm{a}[\AA]$ & $11.422(5)$ & $6.186(1)$ \\
\hline $\mathrm{b}[\AA]$ & $11.628(2)$ & $9.556(1)$ \\
\hline c $[\AA]$ & $22.718(5)$ & $13.169(2)$ \\
\hline$\alpha\left[^{\circ}\right]$ & 90 & $91.958(5)$ \\
\hline$\beta\left[^{\circ}\right]$ & 90 & $99.625(7)$ \\
\hline$\gamma\left[{ }^{\circ}\right]$ & 90 & $106.425(7)$ \\
\hline$V\left[\AA^{3}\right]$ & $3017(2)$ & $733.4(2)$ \\
\hline $\mathrm{Z}$ & 4 & 1 \\
\hline $\mathrm{F}(000)$ & 1160 & 290 \\
\hline$\lambda[\AA]$ & 0.71073 & 0.71073 \\
\hline $\mathrm{T}[\mathrm{K}]$ & 293(2) & 293(2) \\
\hline$\rho_{\text {calc }}\left[\mathrm{Mg} \cdot \mathrm{m}^{-3}\right]$ & 1.188 & 1.222 \\
\hline$\mu\left(\mathrm{Mo}-K_{\alpha}\right)\left[\mathrm{mm}^{-1}\right]$ & 0.152 & 0.156 \\
\hline$\theta$ range $\left[{ }^{\circ} \min -\max \right]$ & $2.50-30.02$ & $2.23-20.81$ \\
\hline data collected & 17895 & 2932 \\
\hline unique data & 8774 & 2932 \\
\hline$R(\mathrm{int})$ & 0.0919 & 0 \\
\hline variable parameters & 354 & 335 \\
\hline obsd.refl. $[\mathrm{I}>2 \sigma(\mathrm{I})]$ & 3652 & 2144 \\
\hline$R$ obsd,. all & $0.0397,0.1705$ & $0.0522,0.0889$ \\
\hline$R_{\mathrm{w}}$ obsd,. all & $0.0852,0.1116$ & $0.1092,0.1270$ \\
\hline$S$ & 0.904 & 1.006 \\
\hline$(\Delta / \sigma)_{\max }$ & 0.001 & 0.000 \\
\hline$(\Delta / \rho)_{\max , \min }\left[\mathrm{e} . \AA^{-3}\right]$ & $0.152,-0.149$ & $0.144,-0.171$ \\
\hline
\end{tabular}




\section{Structure elucidation.}

X-ray diffraction data for $7 \mathbf{b}$ were collected at $293 \mathrm{~K}$ on a Enraf-Nonius CAD4 diffractometer equipped with a graphite monochromator [Mo- $K_{\alpha}$ radiation $\left.(\lambda=0.7107 \AA)\right]$. The data collection and the reduction of data were carried out with the CAD4 Express Enraf-Nonius CAD4 programs package. Data for 7c were collected at $293 \mathrm{~K}$ on a KAPPA CCD Bruker diffractometer with graphite-monochromated Mo- $K_{\alpha}$ radiation using the Bruker AXS Collect Software programs package. All data were corrected for Lorentz-polarization effects. Both the structures were solved by direct methods using SIR-92 $2^{\mathrm{S} 9}$ and refined by full-matrix least-squares methods based on $F^{2}$ with the programs SHELXL-9 $7^{\mathrm{S} 10}$ with anisotropic displacement parameters for all non-hydrogen atoms. Hydrogen atoms were inserted at calculated positions with isotropic parameters constrained to be 1.2 times the $U_{\text {eq }}$ of the carrier atoms (1.5 times for hydrogen atoms of methyl groups) and refined. Molecules were drawn using CAMERON. ${ }^{\text {S11 }}$ All calculations were performed by using the Crystal Structure crystallographic software package WINGX. ${ }^{\text {S12 }}$ 


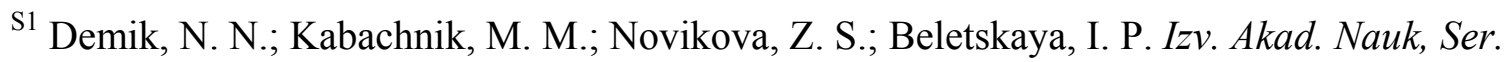
Khim. 1992, 475.

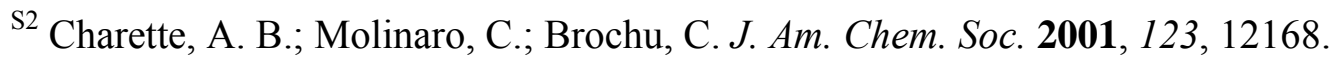

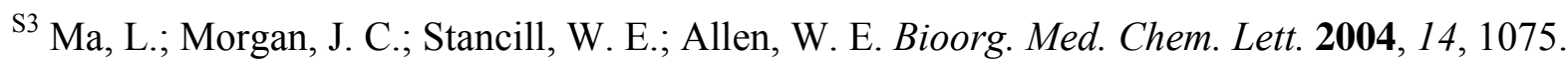

${ }^{\mathrm{S} 4}$ Barbat, J.; Gelas, J.; Horton D. Carbohydr. Res. 1983, 116, 312.

${ }^{\text {S5 }}$ Wang, Y.; He, Q.-F.; Wang, H.-W.; Zhou, X.; Huang, Z.-Y.; Qin, Y. J. Org. Chem. 2006, 71, 1588.

${ }^{\text {S6 }}$ Cignarella, G.; Occelli, E.; Testa, E. J. Med. Chem. 1965, 8, 326.

${ }^{\mathrm{S} 7}$ Whitaker, B. D.; Schmidt, W. F.; Kirk, M. C.; Barnes, S. J. Agric. Food. Chem. 2001, 49 , 3787.

${ }^{\text {S8 }}$ Shuter, E. C.; Duong, H.; Hutton, C. A.; McLeod, M. D. Org. Biomol. Chem. 2007, 5, 3183.

s9 Altomare, A.; Cascarano, G.; Giacovazzo, C.; Guagliardi, A.; Burla, M. C.; Polidori, G.;

Camalli, M. J. Appl. Crystallogr. 1994, 27, 435.

${ }^{\mathrm{S} 10}$ Sheldrick, G. M. SHELXL-97. Program for crystal structure refinement; University of Göttingen, Germany, 1997.

${ }^{\text {S11 }}$ Watkin, D. J.; Prout, C. K.; Pearce L. J. CAMERON; Chemical Crystallography Laboratory, Oxford, UK, 1996.

${ }^{\mathrm{S} 12}$ Farrugia, L. J. J. Appl. Crystallogr. 1999, 32, 837. 